\title{
Assisted reproductive technology and hypertensive disorders of pregnancy: systematic review and meta-analyses
}

\author{
Hui Ju Chih ${ }^{1}$, Flavia T. S. Elias ${ }^{1,2}$, Laura Gaudet ${ }^{1}$ and Maria P. Velez ${ }^{1,3^{*}}$ (D)
}

\begin{abstract}
Background: Hypertensive disorders of pregnancy (HDP) is one of the most common pregnancy complications and causes of maternal morbidity and mortality. Assisted reproductive technology (ART) has been associated with adverse pregnancy outcomes, including HDP. However, the impact of multiple pregnancies, oocyte donation, as well as fresh and frozen embryo transfer needs to be further studied. We conducted a systematic review and metaanalyses to evaluate the association between ART and HDP or preeclampsia relative to spontaneous conception (SC).

Methods: We identified studies from EMBASE, MEDLINE, and Cochrane Library (up to April 8, 2020) and manually using structured search strategies. Cohort studies that included pregnancies after in vitro fertilization (IVF) with or without intracytoplasmic sperm fertilization (ICSI) relative to SC with HDP or preeclampsia as the outcome of interest were included. The control group was women who conceived spontaneously without ART or fertility medications. The pooled results were reported in odds ratios (OR) with $95 \%$ confidence intervals based on random effects models. Numbers needed to harm (NNH) were calculated based on absolute risk differences between exposure and control groups.
\end{abstract}

Results: Eighty-five studies were included after a screening of 1879 abstracts and 283 full text articles. Compared to SC, IVF/ICSI singleton pregnancies (OR 1.70; 95\% Cl 1.60-1.80; $\left.\left.\right|^{2}=80 \%\right)$ and multiple pregnancies (OR 1.34; $95 \% \mathrm{Cl}$ $1.20-1.50 ; I^{2}=76 \%$ ) were both associated with higher odds of HDP. Singleton pregnancies with oocyte donation had the highest odds of HDP out of all groups analyzed (OR 4.42; 95\% Cl 3.00-6.51; $\left.\left.\right|^{2}=83 \%\right)$. Frozen embryo transfer resulted in higher odds of HDP (OR 1.74; 95\% Cl 1.58-1.92; $\left.\left.\right|^{2}=55 \%\right)$ than fresh embryo transfer (OR 1.43; $\left.95 \% \mathrm{Cl} 1.33-1.53 ; I^{2}=72 \%\right)$. The associations between IVF/ICSI pregnancies and SC were similar for preeclampsia. Most interventions had an NNH of 40 to 100, while singleton and multiple oocyte donation pregnancies had particularly low NNH for HDP (16 and 10, respectively).

Conclusions: Our meta-analysis confirmed that IVF/ICSI pregnancies are at higher odds of HDP and preeclampsia than SC, irrespective of the plurality. The odds were especially high in frozen embryo transfer and oocyte donation pregnancies.

\footnotetext{
* Correspondence: maria.velez@queensu.ca

'Department of Obstetrics and Gynecology, Kingston General Hospital,

Queen's University, Victory 476 Stuart St, Kingston, Ontario K7L 2V7, Canada

${ }^{3}$ Department of Public Health Sciences, Queen's University, 62 Fifth Field

Company Lane, Kingston, Ontario K7L 3N6, Canada

Full list of author information is available at the end of the article
}

(c) The Author(s). 2021 Open Access This article is licensed under a Creative Commons Attribution 4.0 International License, which permits use, sharing, adaptation, distribution and reproduction in any medium or format, as long as you give appropriate credit to the original author(s) and the source, provide a link to the Creative Commons licence, and indicate if changes were made. The images or other third party material in this article are included in the article's Creative Commons licence, unless indicated otherwise in a credit line to the material. If material is not included in the article's Creative Commons licence and your intended use is not permitted by statutory regulation or exceeds the permitted use, you will need to obtain permission directly from the copyright holder. To view a copy of this licence, visit http://creativecommons.org/licenses/by/4.0/ The Creative Commons Public Domain Dedication waiver (http://creativecommons.org/publicdomain/zero/1.0/) applies to the data made available in this article, unless otherwise stated in a credit line to the data. 
Keywords: Assisted reproductive technology, In vitro fertilization, Intracytoplasmic sperm injection, Hypertensive disorders of pregnancy, Preeclampsia, Frozen embryo transfer, Fresh embryo transfer, Oocyte donation, Metaanalysis

\section{Background}

In 2010, 48.5 million couples worldwide were estimated to be affected by infertility [1]. The use of in vitro fertilization (IVF) and other assisted reproductive technologies (ART) is expanding rapidly, accounting for more than seven million births worldwide [2]. The advancement of treatments and changes in protocols have also reshaped the landscape of fertility practice in recent years. For example, intracytoplasmic sperm injection (ICSI) is mainly indicated for male factor infertility or poor response to IVF [3]; cryopreservation has led to the rise of frozen embryo transfer (FET), which expands the scope of treatment and decreases the risk of ovarian hyperstimulation syndrome [4]; finally, oocyte donation (OD) allows women with decreased ovarian reserve or ovarian failure to achieve pregnancy [5].

While ART continues to benefit many couples around the world, it may be associated with adverse outcomes, including hypertensive disorders of pregnancy (HDP) [6-10]. HDP, including gestational hypertension and preeclampsia, occur in approximately $12-22 \%$ of all pregnancies and is associated with significant maternal and prenatal morbidity and mortality [11]. Preeclampsia is associated with a wide range of complications related to microangiopathy, vasoconstriction, and malperfusion. Women with a history of preeclampsia also continue to be at a high risk for cardiovascular disease, chronic kidney disease, and cardiovascular mortality even after pregnancy [12]. The pregnancy and postpartum complications as well as high mortality rates highlight the importance of prevention and early detection of HDP.

Although previous meta-analyses have shown that ART is associated with an increased risk of preeclampsia, the underlying mechanism is not well understood $[13,14]$. Many included studies were based on singleton pregnancies or mixed cohorts, while studies specifically comparing the risk of HDP in ART and spontaneous multiple pregnancies often yielded inconsistent results $[9,15]$. The types of ART and treatment protocols also appear to play a role in differences in maternal and perinatal outcomes [16]. For example, recent meta-analysis by Rogue et al. showed that FET is associated with a higher rate of low birth weight and preeclampsia when compared to fresh ET [17]. Currently, it remains unclear whether the differences in pregnancy outcomes, including HDP, were due to maternal factors, the procedure itself, or both. With the increasing number of literature over the past decade and changes in protocols, there is a need for an updated and comprehensive review of IVF/ ICSI pregnancies in consideration of patient and treatment factors.

Our systematic review and meta-analysis aim to understand whether IVF/ICSI pregnancies are associated with increased odds of HDP and preeclampsia in comparison to spontaneous conception (SC); furthermore, we aim to understand whether the odds differ depending on types of procedure. Together, this review may inform clinical recommendations for women planning to achieve pregnancy through IVF/ICSI.

\section{Methods \\ Search strategy}

The study follows the Preferred Reporting Items for Systematic Reviews and Meta-Analysis (PRISMA) checklist (Supplementary information, Additional file 1) and the protocol was registered and available on Open Science Framework (DOI: https://osf.io/562jr/). A search strategy was developed under the support of a research librarian to identify studies evaluating the incidence of HDP and/ or preeclampsia in IVF or ICSI pregnancies compared to $\mathrm{SC}$ (Additional file 2). MeSH terms and selection criteria were based on the Patient, Intervention, Comparison and Outcome statement. Cohort studies published up to April 8, 2020 were retrieved from Medline, Embase, and Cochrane Central Register of controlled Trials using the OVID platform. A manual search of previously published systematic reviews and meta-analysis was also conducted to identify other eligible studies.

\section{Selection of studies}

Both abstract and full text screening were performed by two reviewers (HC, FTSE). In the first screening, articles were selected based on titles and abstracts. The second screening involved full-text reviews, where studies were evaluated based on a set of eligibility criteria. Any conflict was resolved by consensus or the involvement of a third team member (MPV).

Studies that compared pregnancies after IVF or ICSI and SC with HDP or preeclampsia as the outcome of interest were included. The control group consisted of women who conceived spontaneously without the use of ART or fertility medications. The exposure group consisted of singleton or multiple IVF/ICSI pregnancies. Non-randomized studies in the form of prospective and retrospective cohort studies were of interest; other study designs such as review articles, randomized control 
trials, case-control studies, conference abstracts, and case reports were excluded. Studies were excluded if they were not in English, French, Portuguese, or Chinese, included patients undergoing ART or fertility treatments other than IVF/ICSI, did not specify the type of ART used, or did not clearly separate patients into singleton or multiple pregnancies. Studies that included a subgroup of women (e.g. advanced maternal age, obesity) were not included in the general singleton and multiple gestation analyses as they were not representative of the general population. However, they were included in sub-analyses for type of embryo transfer (fresh embryo transfer (fresh ET) or FET) and OD. For studies with overlapping cohorts, where the same database was used for analyses, only the most recent study was included in the meta-analysis. A complete list of excluded studies after full text screening with their respective reasons of exclusion may be found in Additional file 3.

Outcomes of interest included HDP and preeclampsia. Hypertensive disorders of pregnancy describe any hypertensive effects that is observed during pregnancy, including pre-existing hypertension, gestational hypertension and preeclampsia. Preeclampsia was defined as hypertension that develops for the first time after 20 weeks of gestation with one or more of the following: proteinuria, adverse conditions, or severe complications [18].

\section{Data extraction and quality assessment}

Data was extracted manually and entered into an Excel spreadsheet by a reviewer (HC). The following characteristics of each study were collected: authorship, year of publication, country, study design, search database, time period of the cohort, matching factors, statistical analysis, outcome of interest, definition of outcome, mean maternal age, mean BMI, number of patients with chronic hypertension, type of ART, type of infertility, source of oocyte, method of embryo transfer, sample size, and crude data. If needed, percentages of HDP and preeclampsia were converted to crude data based on the sample size. Study quality was assessed using the Newcastle-Ottawa Scale for Cohort Studies [19]. Each study was scored out of nine based on eight items across three domains: the selection of study groups (4 items), comparability of groups ( 1 item), and ascertainment of exposure or outcome of interest in cohort studies, respectively (3 items). It was then determined to have either high quality ( 8 or 9 ), moderate quality (6 or 7 ), or low quality (less than 5 ) based on the total NOS score. A second reviewer (FTSE) reviewed all data extraction and quality assessment performed.

\section{Statistical analysis}

The meta-analyses were performed using Review Manager (RevMan) version 5.4. Cohort studies were included in the general meta-analyses by plurality. Studies that explicitly excluded ICSI pregnancies and those that included ICSI pregnancies only were included in the IVF and ICSI sub-analyses, respectively. In addition, separate analyses on fresh ET, FET, and OD were also conducted. Results were reported as odds ratios with corresponding 95\% confidence intervals based on random effects models, which assumed heterogeneity of the data. The Mantel-Haenszel method was used to calculate overall odds ratios. Statistical significance was determined by a $P$ value of equal or less than 0.05 . Numbers needed to harm $(\mathrm{NNH})$, which represented the number of patients needed to undergo IVF/ICSI for one patient to receive harm, were calculated based on the absolute risk differences between exposure and control groups [20]. Sensitivity analysis was carried out by removing one study at a time to assess the effect of the study on the results. If the measure of association without the chosen study fell outside of the confidence interval, the study was said to have a significant influence [21]. I-squared $\left(\mathrm{I}^{2}\right)$ test was used to evaluate heterogeneity, with an $\mathrm{I}^{2}$ value of greater than $50 \%$ being considered as high heterogeneity [22]. Risk of publication bias was evaluated using funnel plots if the meta-analysis included 10 or more studies [23].

\section{Results \\ Study selection}

Our search strategy identified 2674 studies and 1879 citations were eligible for abstract and title screening after removing duplicates. Finally, 85 studies met the inclusion criteria and were included in the meta-analysis (Fig. 1). Eight studies had overlapping cohorts with more recently published studies and thus were excluded (Additional file 3).

\section{Characteristics of included studies}

The characteristics of all included studies, which involved 405,920 IVF/ICSI pregnancies and 8,122,210 SC, may be found in Table 1. The sizes of the exposure (IVF/ICSI) and control (SC) groups ranged from 19 to 83,582 pregnancies and from 21 to $1,382,311$ pregnancies respectively. Out of the 85 studies included, 21 were population-based cohort studies conducted in Canada [39, 40, 88], Denmark [64], Finland [55, 78], Israel [73, 85], China [105], Japan [69], Netherlands [31], Norway [94], Slovenia [52], Sweden [43, 44, 71, 83], and the United States [62, 63]. Two studies were conducted across multiple European countries [75, 101]. Fifty-one studies looked at the incidences of preeclampsia or HDP in IVF/ICSI singleton pregnancies in comparison to SC, while 41 studies investigated the outcomes of multiple pregnancies in particular (Table 1). Based on their respective NOS scores, 15 studies had a high 


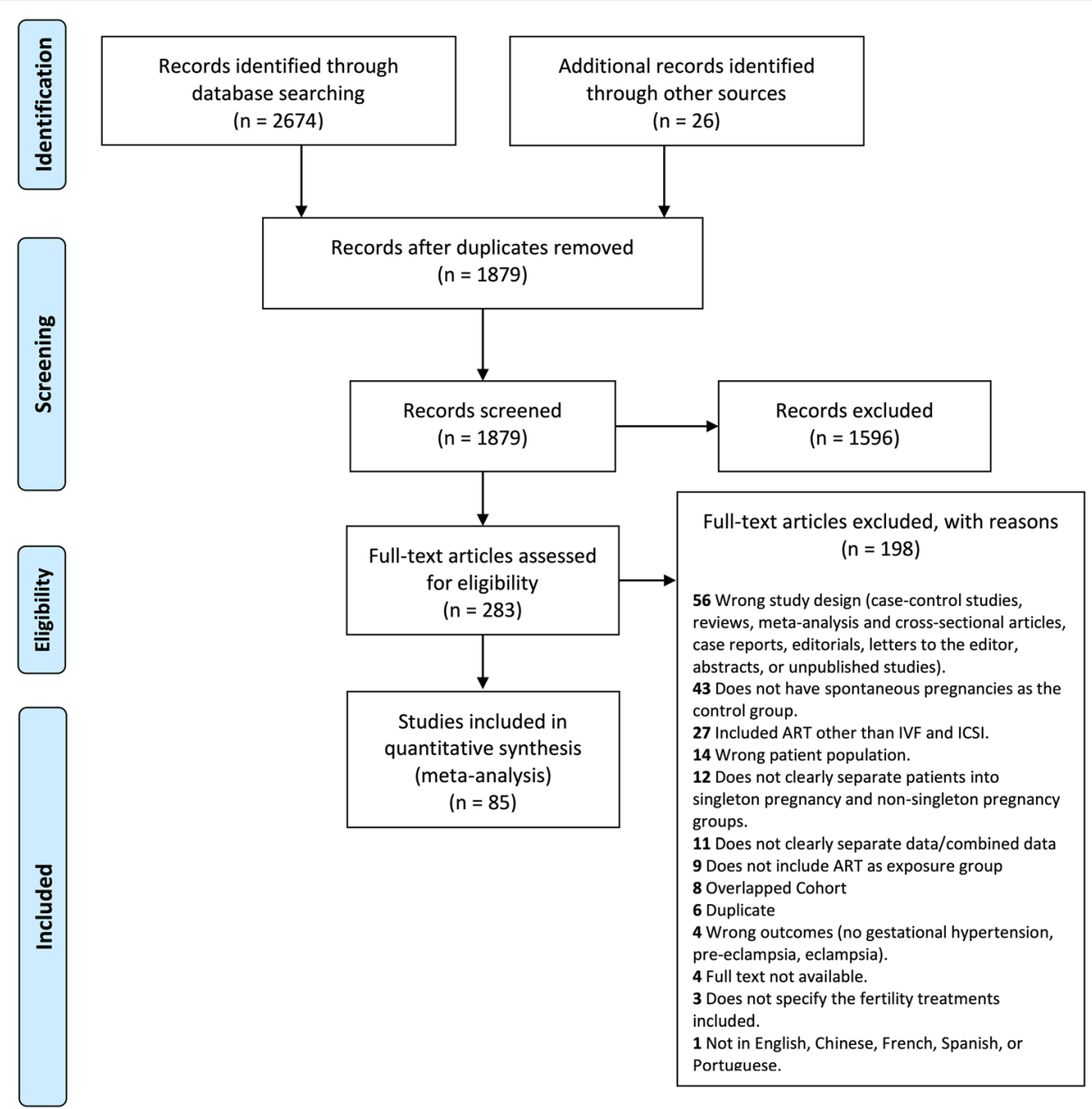

Fig. 1 PRISMA Flowchart. Flow diagram for study identification and inclusion according to the Preferred Reporting Items for Systematic Reviews and Meta-Analyses (PRISMA) guidelines

methodological quality, 61 studies had a moderate quality, and 9 had a low quality (Table 1, Additional file 4). Thirty-three studies were matched cohort studies using varying factors such as maternal age, birth year, parity, socioeconomic status, location (Table 1). Eight studies used chronic hypertension to adjust the comparability between exposure and control groups [65, $70,75,81,88,97,100,108]$. One study calculated propensity scores that account for 27 maternal and paternal variables [100].

\section{Specific outcomes}

\section{IVF/ICSI singleton pregnancies}

Fifty-one studies including 268,166 pregnancies in the IVF/ICSI group and 7.7 million pregnancies in the SC group were included in the analysis of HDP. The overall odds ratio (OR) was 1.70 (95\% CI 1.60-1.80) with high heterogeneity $\left(\mathrm{I}^{2}=80 \%\right)$ (Fig. 2$)$. Almost all studies were of high or moderate quality according to their NOS scores; four were categorized as low quality. A separate analysis of 5 studies that included only IVF pregnancies yielded consistent findings with an OR of 1.55 (95\% CI 1.23-1.94; $\mathrm{I}^{2}=90 \%$ ) (Fig. 3).

Of those studies above, 28 studies specifically reported the incidence of preeclampsia, resulting in a sample size of 118,787 pregnancies in the IVF/ICSI group and 4.5 million pregnancies in the SC group. Cumulative incidences found that the IVF/ICSI group had significantly higher odds of preeclampsia than the SC group with an OR of 1.59 (95\% CI 1.46-1.74) with high heterogeneity $\left(\mathrm{I}^{2}=70 \%\right.$ ) (Additional file 5$)$. Twenty-seven studies were classified as high or moderate quality, and one study received an NOS score of low quality. Half of the included studies matched the control group with IVF/ICSI group by maternal factors such as age or parity. Only one study studying preeclampsia explicitly excluded ICSI pregnancies and therefore a sub-analysis for IVF could not be conducted.

The sub-analysis of nine studies that included ICSI pregnancies only found that this type of procedure had a higher rate of HDP in comparison to SC. The resulting OR was $1.52\left(95 \%\right.$ CI $\left.1.28-1.80 ; \mathrm{I}^{2}=75 \%\right)$ (Fig. 3). In the 
Table 1 Characteristics of 78 included cohort studies. $S=$ Singleton pregnancy, $M=$ Multiple pregnancy

\begin{tabular}{|c|c|c|c|c|c|c|c|}
\hline $\begin{array}{l}\text { First author, } \\
\text { Publication } \\
\text { year, Country }\end{array}$ & $\begin{array}{l}\text { Type of } \\
\text { cohort }\end{array}$ & $\begin{array}{l}\text { Years of the } \\
\text { cohort }\end{array}$ & Matching factors & Comparison groups & $\begin{array}{l}\text { Pregnancies } \\
\text { conceived } \\
\text { by IVF/ICSI } \\
\text { (n) }\end{array}$ & $\begin{array}{l}\text { Spontaneous } \\
\text { pregnancies } \\
\text { (n) }\end{array}$ & $\begin{array}{l}\text { NOS } \\
\text { Score }\end{array}$ \\
\hline $\begin{array}{l}\text { Agarwal, 2005, } \\
\text { Singapore [24] }\end{array}$ & $\begin{array}{l}\text { Hospital- } \\
\text { based } \\
\text { Retrospective }\end{array}$ & 1998-1999 & $\begin{array}{l}\text { Maternal age, sex, date of } \\
\text { delivery, race, plurality and } \\
\text { parity }\end{array}$ & IVF/ICSI, ICSI alone & $\begin{array}{l}\text { (S) } 41 \\
\text { (M) } 35\end{array}$ & $\begin{array}{l}\text { (S) } 147 \\
\text { (M) } 114\end{array}$ & 7 \\
\hline $\begin{array}{l}\text { Ai, 2005, China } \\
\text { [25] }\end{array}$ & $\begin{array}{l}\text { Hospital- } \\
\text { based } \\
\text { Retrospective }\end{array}$ & 1998-2004 & No & IVF/ICSI & (M) 47 & (M) 98 & 6 \\
\hline $\begin{array}{l}\text { Apantaku, 2008, } \\
\text { UK [26] }\end{array}$ & $\begin{array}{l}\text { Hospital- } \\
\text { based } \\
\text { Retrospective }\end{array}$ & 1999-2004 & Maternal age, parity & IVF/ICSI & (S) 88 & (S) 88 & 8 \\
\hline $\begin{array}{l}\text { Aydin, 2016, } \\
\text { Turkey [27] }\end{array}$ & $\begin{array}{l}\text { Hospital- } \\
\text { based } \\
\text { Retrospective }\end{array}$ & $2007-2010$ & Maternal age & IVF/ICSI & (M)137 & (M) 133 & 8 \\
\hline $\begin{array}{l}\text { Barda, 2017, } \\
\text { Israel [28] }\end{array}$ & $\begin{array}{l}\text { Hospital- } \\
\text { based } \\
\text { Retrospective }\end{array}$ & 2009-2015 & No & IVF/ICSI & (M) 449 & (M) 259 & 6 \\
\hline $\begin{array}{l}\text { Barua, 2016, } \\
\text { Australia [29] }\end{array}$ & $\begin{array}{l}\text { Hospital- } \\
\text { based } \\
\text { Retrospective }\end{array}$ & $2007-2010$ & No & IVF/ICSI, ICSI alone & (S) 470 & (S) 48654 & 6 \\
\hline $\begin{array}{l}\text { Beltran Anzola, } \\
\text { 2019, France [30] }\end{array}$ & $\begin{array}{l}\text { Hospital- } \\
\text { based } \\
\text { Retrospective }\end{array}$ & 1995-2015 & $\begin{array}{l}\text { Maternal age, exact year of } \\
\text { birth, parity, sex }\end{array}$ & $\begin{array}{l}\text { IVF/ICSI, Fresh } \\
\text { embryo transfer, } \\
\text { Frozen embryo } \\
\text { transfer }\end{array}$ & (S) 2327 & (S) 6981 & 7 \\
\hline $\begin{array}{l}\text { Bensdorp, 2016, } \\
\text { Netherlands [31] } \\
\text { (a) }\end{array}$ & $\begin{array}{l}\text { Population- } \\
\text { based } \\
\text { Retrospective }\end{array}$ & 2000-2012 & $\begin{array}{l}\text { Zygosity, parity, } \\
\text { socioeconomic status, } \\
\text { conception method }\end{array}$ & IVF/ICSI, ICSI alone & (M) 2437 & (M) 3276 & 7 \\
\hline $\begin{array}{l}\text { Beyer, 2016, } \\
\text { Germany [32] }\end{array}$ & $\begin{array}{l}\text { Hospital- } \\
\text { based } \\
\text { Retrospective }\end{array}$ & $\begin{array}{l}\text { N/A (13-year } \\
\text { period) }\end{array}$ & No & $\begin{array}{l}\text { IVF/ICSI, Fresh } \\
\text { embryo transfer, } \\
\text { Frozen embryo } \\
\text { transfer }\end{array}$ & (S) 467 & (S) 6417 & 6 \\
\hline $\begin{array}{l}\text { Carbone, 2011, } \\
\text { UK [33] }\end{array}$ & $\begin{array}{l}\text { Hospital- } \\
\text { based } \\
\text { Prospective }\end{array}$ & 2006-2009 & No & IVF/ICSI & (S) 426 & (S) 26538 & 7 \\
\hline $\begin{array}{l}\text { Caserta, 2008, } \\
\text { Italy [34] }\end{array}$ & $\begin{array}{l}\text { Hospital- } \\
\text { based } \\
\text { Prospective }\end{array}$ & 2004-2006 & $\begin{array}{l}\text { Parity, age, height, weight, } \\
\text { ethnic origin, smoking, history } \\
\text { of infertility }\end{array}$ & IVF/ICSI, ICSI alone & (S) 364 & (S) 304 & 3 \\
\hline $\begin{array}{l}\text { Caserta, 2014, } \\
\text { Italy [35] }\end{array}$ & $\begin{array}{l}\text { Hospital- } \\
\text { based } \\
\text { Retrospective }\end{array}$ & $2007-2011$ & No & IVF/ICSI & (M) 138 & (M) 207 & 7 \\
\hline $\begin{array}{l}\text { Choi, 2006, Korea } \\
\text { [36] }\end{array}$ & $\begin{array}{l}\text { Hospital- } \\
\text { based } \\
\text { Retrospective }\end{array}$ & 1994-2003 & No & IVF/ICSI & (M) 190 & (M) 347 & 6 \\
\hline $\begin{array}{l}\text { Daniel, 2000, } \\
\text { Israel [37] }\end{array}$ & $\begin{array}{l}\text { Hospital- } \\
\text { based } \\
\text { Retrospective }\end{array}$ & 1996-1997 & No & IVF/ICSI & (M) 104 & (M) 121 & 7 \\
\hline $\begin{array}{l}\text { Dayan, 2015, } \\
\text { Canada [38] }\end{array}$ & $\begin{array}{l}\text { Hospital- } \\
\text { based } \\
\text { Retrospective }\end{array}$ & $2001-2008$ & No & IVF/ICSI & (S) 326 & (S) 9175 & 7 \\
\hline $\begin{array}{l}\text { Dayan, 2016, } \\
\text { Canada [39] }\end{array}$ & $\begin{array}{l}\text { Population- } \\
\text { based } \\
\text { Retrospective }\end{array}$ & 2006-2012 & No & IVF/ICSI & (S) 5371 & (S) 795997 & 7 \\
\hline $\begin{array}{l}\text { Dayan, 2018, } \\
\text { Canada [40] }\end{array}$ & $\begin{array}{l}\text { Population- } \\
\text { based } \\
\text { Retrospective }\end{array}$ & 2013-2014 & No & IVF/ICSI & (S) 1596 & (S) 112813 & 6 \\
\hline $\begin{array}{l}\text { Deltombe- } \\
\text { Bodart, 2017, } \\
\text { France [41] }\end{array}$ & $\begin{array}{l}\text { Hospital- } \\
\text { based } \\
\text { Retrospective }\end{array}$ & 1997-2014 & No & IVF/ICSI, ICSI alone & (M) 360 & (M) 986 & 6 \\
\hline
\end{tabular}


Table 1 Characteristics of 78 included cohort studies. S = Singleton pregnancy, M= Multiple pregnancy (Continued)

\begin{tabular}{|c|c|c|c|c|c|c|c|}
\hline $\begin{array}{l}\text { First author, } \\
\text { Publication } \\
\text { year, Country }\end{array}$ & $\begin{array}{l}\text { Type of } \\
\text { cohort }\end{array}$ & $\begin{array}{l}\text { Years of the } \\
\text { cohort }\end{array}$ & Matching factors & Comparison groups & $\begin{array}{l}\text { Pregnancies } \\
\text { conceived } \\
\text { by IVF/ICSI } \\
\text { (n) }\end{array}$ & $\begin{array}{l}\text { Spontaneous } \\
\text { pregnancies } \\
\text { (n) }\end{array}$ & $\begin{array}{l}\text { NOS } \\
\text { Score }\end{array}$ \\
\hline $\begin{array}{l}\text { Dior, 2018, Israel } \\
\text { [42] }\end{array}$ & $\begin{array}{l}\text { Hospital- } \\
\text { based } \\
\text { Retrospective }\end{array}$ & 1995-2012 & No & Oocyte Donation & (S) 135 & (S) 270 & 7 \\
\hline $\begin{array}{l}\text { Elenis, 2015, } \\
\text { Sweden [43] }\end{array}$ & $\begin{array}{l}\text { Population- } \\
\text { based } \\
\text { Retrospective }\end{array}$ & $2005-2008$ & Age & $\begin{array}{l}\text { IVF/ICSI, Oocyte } \\
\text { Donation }\end{array}$ & (S) 139 & (M) 150 & 7 \\
\hline $\begin{array}{l}\text { Ernstad, 2019, } \\
\text { Sweden [44] }\end{array}$ & $\begin{array}{l}\text { Population- } \\
\text { based } \\
\text { Retrospective }\end{array}$ & $2005-2015$ & No & $\begin{array}{l}\text { IVF/ICSI, Fresh } \\
\text { embryo transfer }\end{array}$ & (S) 34091 & (S) 1127566 & 6 \\
\hline $\begin{array}{l}\text { Fan, 2013, China } \\
\text { [45] }\end{array}$ & $\begin{array}{l}\text { Hospital- } \\
\text { based } \\
\text { Retrospective }\end{array}$ & 2010-2013 & No & IVF/ICSI, ICSI alone & (M) 162 & (M) 213 & 7 \\
\hline $\begin{array}{l}\text { Farhi, 2013, Israel } \\
\text { [46] }\end{array}$ & $\begin{array}{l}\text { Hospital- } \\
\text { based } \\
\text { Prospective }\end{array}$ & $2006-2008$ & No & IVF/ICSI, ICSI alone & (S) 509 & (S) 587 & 5 \\
\hline $\begin{array}{l}\text { Geipel, 2001, } \\
\text { Germany [47] }\end{array}$ & $\begin{array}{l}\text { Hospital- } \\
\text { based } \\
\text { Retrospective }\end{array}$ & 1995-1999 & Maternal age, parity, plurality & IVF/ICSI, ICSI alone & $\begin{array}{l}\text { (S) } 114 \\
\text { (M) } 32\end{array}$ & $\begin{array}{l}\text { (S) } 114 \\
\text { (M) } 32\end{array}$ & 8 \\
\hline $\begin{array}{l}\text { Gocmen, 2015, } \\
\text { Turkey [48] }\end{array}$ & $\begin{array}{l}\text { Hospital- } \\
\text { based } \\
\text { Retrospective }\end{array}$ & $2011-2014$ & No & IVF/ICSI & (M) 19 & (M) 65 & 8 \\
\hline $\begin{array}{l}\text { Gojnic, 2005, } \\
\text { Serbia [49] }\end{array}$ & N/A & N/A & Age, education, parity & IVF/ICSI & (M) 120 & (M) 120 & 2 \\
\hline $\begin{array}{l}\text { Hessami, 2020, } \\
\text { Iran [50] }\end{array}$ & $\begin{array}{l}\text { Hospital- } \\
\text { based } \\
\text { Retrospective }\end{array}$ & 2013-2018 & No & IVF/ICSI & (M) 202 & (M) 449 & 6 \\
\hline $\begin{array}{l}\text { Howe, 1990, US } \\
\text { [51] }\end{array}$ & $\begin{array}{l}\text { Hospital- } \\
\text { based } \\
\text { Retrospective }\end{array}$ & $\begin{array}{l}\text { N/A (first } 100 \\
\text { clinical } \\
\text { pregnancies } \\
\text { conceived in the } \\
\text { IVF program) }\end{array}$ & $\begin{array}{l}\text { Age, race, parity, pre-existing } \\
\text { medical problem, DES expos- } \\
\text { ure, insurance status }\end{array}$ & IVF/ICSI & (S) 54 & (S)54 & 7 \\
\hline $\begin{array}{l}\text { Jancar, 2018, } \\
\text { Slovenia [52] }\end{array}$ & $\begin{array}{l}\text { Population- } \\
\text { based } \\
\text { Retrospective }\end{array}$ & $2002-2015$ & No & IVF/ICSI & (S) 5837 & (S) 261881 & 7 \\
\hline $\begin{array}{l}\text { Jeve, 2016, UK } \\
\text { [53] }\end{array}$ & $\begin{array}{l}\text { Hospital- } \\
\text { based } \\
\text { Retrospective }\end{array}$ & 2007-2014 & Age & $\begin{array}{l}\text { IVF/ICSI, Oocyte } \\
\text { donation }\end{array}$ & (S) 90 & (S) 45 & 8 \\
\hline $\begin{array}{l}\text { Katalinic, 2004, } \\
\text { Germany [54] }\end{array}$ & $\begin{array}{l}\text { Hospital- } \\
\text { based } \\
\text { Prospective }\end{array}$ & $\begin{array}{l}\text { 1998-2000; 1993- } \\
2001 \text { (control) }\end{array}$ & No & $\begin{array}{l}\text { IVF/ICSI, ICSI alone, } \\
\text { Fresh embryo } \\
\text { transfer }\end{array}$ & $\begin{array}{l}\text { (S) } 2055 \\
\text { (M) } 632\end{array}$ & $\begin{array}{l}\text { (S) } 7861 \\
\text { (M) } 77\end{array}$ & 6 \\
\hline $\begin{array}{l}\text { Koivurova, 2002, } \\
\text { Finland [55] }\end{array}$ & $\begin{array}{l}\text { Population- } \\
\text { based } \\
\text { Retrospective }\end{array}$ & 1990-1995 & $\begin{array}{l}\text { Sex of the child, birth year, } \\
\text { area of residence, parity, } \\
\text { maternal age, social class } \\
\text { (defined by occupation) }\end{array}$ & $\begin{array}{l}\text { IVF/ICSI, Fresh } \\
\text { embryo transfer }\end{array}$ & $\begin{array}{l}\text { (S) } 153 \\
\text { (M) } 62\end{array}$ & $\begin{array}{l}\text { (S) } 580 \\
\text { (M) } 82\end{array}$ & 7 \\
\hline $\begin{array}{l}\text { Korosec, 2016, } \\
\text { Slovenia [56] }\end{array}$ & $\begin{array}{l}\text { Hospital- } \\
\text { based } \\
\text { Retrospective }\end{array}$ & 2004-2011 & Age, parity, hospital & $\begin{array}{l}\text { IVF/ICSI, Fresh } \\
\text { embryo transfer, } \\
\text { Frozen embryo } \\
\text { transfer }\end{array}$ & (S) 1127 & (S) 3381 & 7 \\
\hline $\begin{array}{l}\text { Kouhkan, 2018, } \\
\text { Iran [57] }\end{array}$ & $\begin{array}{l}\text { Hospital- } \\
\text { based } \\
\text { Prospective }\end{array}$ & 2014-2017 & No & IVF/ICSI & (S) 260 & (S) 314 & 7 \\
\hline $\begin{array}{l}\text { Kuivasaari- } \\
\text { Pirinen, 2012, } \\
\text { Finland [58] }\end{array}$ & $\begin{array}{l}\text { Hospital- } \\
\text { based } \\
\text { Retrospective }\end{array}$ & 1996-2007 & No & IVF/ICSI & (S) 255 & (S) 26870 & 7 \\
\hline $\begin{array}{l}\text { Lee, 2015, US } \\
\text { [59] }\end{array}$ & $\begin{array}{l}\text { Hospital- } \\
\text { based }\end{array}$ & $2007-2009$ & No & IVF/ICSI & (S) 108 & (S) 2284 & 7 \\
\hline
\end{tabular}


Table 1 Characteristics of 78 included cohort studies. S = Singleton pregnancy, M= Multiple pregnancy (Continued)

\begin{tabular}{|c|c|c|c|c|c|c|c|}
\hline $\begin{array}{l}\text { First author, } \\
\text { Publication } \\
\text { year, Country }\end{array}$ & $\begin{array}{l}\text { Type of } \\
\text { cohort }\end{array}$ & $\begin{array}{l}\text { Years of the } \\
\text { cohort }\end{array}$ & Matching factors & Comparison groups & $\begin{array}{l}\text { Pregnancies } \\
\text { conceived } \\
\text { by IVF/ICSI } \\
\text { (n) }\end{array}$ & $\begin{array}{l}\text { Spontaneous } \\
\text { pregnancies } \\
\text { (n) }\end{array}$ & $\begin{array}{l}\text { NOS } \\
\text { Score }\end{array}$ \\
\hline & Retrospective & & & & & & \\
\hline $\begin{array}{l}\text { Lei, 2019, China } \\
\text { [60] }\end{array}$ & $\begin{array}{l}\text { Hospital- } \\
\text { based } \\
\text { Retrospective }\end{array}$ & 2013-2015 & No & IVF/ICSI & $\begin{array}{l}\text { (S) } 1453 \\
\text { (M) } 803\end{array}$ & $\begin{array}{l}\text { (S) } 6667 \\
\text { (M) } 101\end{array}$ & 6 \\
\hline $\begin{array}{l}\text { Li, 2015, China } \\
\text { [61] }\end{array}$ & $\begin{array}{l}\text { Hospital- } \\
\text { based } \\
\text { Retrospective }\end{array}$ & 2009-2011 & No & IVF/ICSI & (M) 108 & (M) 144 & 6 \\
\hline $\begin{array}{l}\text { Luke, 2019, US } \\
\text { [62] }\end{array}$ & $\begin{array}{l}\text { Population- } \\
\text { based } \\
\text { Retrospective }\end{array}$ & $\begin{array}{l}\text { 2004-2013 } \\
\text { (depending on } \\
\text { states) }\end{array}$ & No & $\begin{array}{l}\text { IVF/ICSI, Oocyte } \\
\text { donation }\end{array}$ & (M) 58,920 & (M) 34,033 & 7 \\
\hline $\begin{array}{l}\text { Luke, 2020, US } \\
\text { [63] }\end{array}$ & $\begin{array}{l}\text { Population- } \\
\text { based } \\
\text { Retrospective }\end{array}$ & $\begin{array}{l}\text { 2004-2013 } \\
\text { (depending on } \\
\text { states) }\end{array}$ & No & $\begin{array}{l}\text { IVF/ICSI, Fresh } \\
\text { embryo transfer, } \\
\text { Frozen embryo } \\
\text { transfer, Oocyte } \\
\text { donation }\end{array}$ & (S) 83582 & (S) 1382311 & 6 \\
\hline $\begin{array}{l}\text { Malchau, 2013, } \\
\text { Denmark [64] }\end{array}$ & $\begin{array}{l}\text { Population- } \\
\text { based } \\
\text { Retrospective }\end{array}$ & 1995-2010 & Date and year of birth & $\begin{array}{l}\text { IVF/ICSI, ICSI alone, } \\
\text { Oocyte donation }\end{array}$ & $\begin{array}{l}\text { (S) } 15741 \\
\text { (M) } 8564\end{array}$ & $\begin{array}{l}\text { (S) } 31010 \\
\text { (M) } 25,012\end{array}$ & 7 \\
\hline $\begin{array}{l}\text { Martinez-Varea, } \\
\text { 2015, Spain [65] }\end{array}$ & $\begin{array}{l}\text { Hospital- } \\
\text { based } \\
\text { Prospective }\end{array}$ & N/A & No & $\begin{array}{l}\text { IVF/ICSI, Oocyte } \\
\text { donation }\end{array}$ & (S) 50 & (S) 25 & 6 \\
\hline $\begin{array}{l}\text { Meyer, 2020, } \\
\text { Israel [66] }\end{array}$ & $\begin{array}{l}\text { Hospital- } \\
\text { based } \\
\text { Retrospective }\end{array}$ & 2011-2018 & Age & Oocyte donation & (S) 159 & (S) 73 & 7 \\
\hline $\begin{array}{l}\text { Mohammed, } \\
\text { 2012, Qatar [67] }\end{array}$ & $\begin{array}{l}\text { Hospital- } \\
\text { based } \\
\text { Retrospective }\end{array}$ & 2002-2011 & No & IVF/ICSI & (M) 145 & (M) 175 & 7 \\
\hline $\begin{array}{l}\text { Moini, 2012, Iran } \\
\text { [68] }\end{array}$ & $\begin{array}{l}\text { Hospital- } \\
\text { based } \\
\text { Prospective }\end{array}$ & 2008-2010 & No & IVF/ICSI, ICSI alone & (M) 230 & (M) 170 & 6 \\
\hline $\begin{array}{l}\text { Nagata, 2019, } \\
\text { Japan [69] }\end{array}$ & $\begin{array}{l}\text { Population- } \\
\text { based } \\
\text { Retrospective }\end{array}$ & 2011-2014 & No & IVF/ICSI, ICSI alone & $\begin{array}{l}\text { (S) } 2993 \\
\text { (M) } 129\end{array}$ & $\begin{array}{l}\text { (S) } 88873 \\
\text { (M) } 625\end{array}$ & 7 \\
\hline $\begin{array}{l}\text { Nassar, 2003, } \\
\text { Lebanon [70] }\end{array}$ & $\begin{array}{l}\text { Hospital- } \\
\text { based } \\
\text { Retrospective }\end{array}$ & 1995-2000 & Age, parity & IVF/ICSI & (M) 56 & (M) 112 & 9 \\
\hline $\begin{array}{l}\text { Nejdet, 2016, } \\
\text { Sweden [71] }\end{array}$ & $\begin{array}{l}\text { Population- } \\
\text { based } \\
\text { Retrospective }\end{array}$ & 2003-2012 & No & $\begin{array}{l}\text { IVF/ICSI, Fresh } \\
\text { embryo transfer, } \\
\text { Frozen embryo } \\
\text { transfer, Oocyte } \\
\text { donation }\end{array}$ & (S) 27084 & (S) 999804 & 7 \\
\hline $\begin{array}{l}\text { Ochsenkuehn, } \\
\text { 2003, Germany } \\
\text { [72] }\end{array}$ & $\begin{array}{l}\text { Hospital- } \\
\text { based } \\
\text { Retrospective }\end{array}$ & 1991-1996 & $\begin{array}{l}\text { Maternal age, gestational age, } \\
\text { parity }\end{array}$ & IVF/ICSI & $\begin{array}{l}\text { (S) } 163 \\
\text { (M) } 65\end{array}$ & $\begin{array}{l}\text { (S) } 322 \\
\text { (M) } 78\end{array}$ & 8 \\
\hline $\begin{array}{l}\text { Okby, 2018, Israel } \\
\text { [73] }\end{array}$ & $\begin{array}{l}\text { Population- } \\
\text { based } \\
\text { Retrospective }\end{array}$ & 1988-2010 & No & IVF/ICSI & (M) 465 & (M) 3053 & 8 \\
\hline $\begin{array}{l}\text { Olivennes, 1993, } \\
\text { France [74] }\end{array}$ & $\begin{array}{l}\text { Hospital- } \\
\text { based } \\
\text { Retrospective }\end{array}$ & 1987-1989 & No & IVF/ICSI & (S) 162 & (S) 5096 & 6 \\
\hline $\begin{array}{l}\text { Opdahl, 2015, } \\
\text { Sweden, } \\
\text { Denmark, } \\
\text { Norway [75] }\end{array}$ & $\begin{array}{l}\text { Population- } \\
\text { based } \\
\text { Retrospective }\end{array}$ & 1988-2007 & Parity, birth year & IVF/ICSI & $\begin{array}{l}\text { (S) } 47088 \\
\text { (M) } 10,918\end{array}$ & $\begin{array}{l}\text { (S) } 268599 \\
\text { (M) } 46,674\end{array}$ & 9 \\
\hline $\begin{array}{l}\text { Poikkeus, 2007, } \\
\text { Finland [76] }\end{array}$ & $\begin{array}{l}\text { Hospital- } \\
\text { based }\end{array}$ & 1997-2003 & Year, place of residence & $\begin{array}{l}\text { IVF/ICSI, Fresh } \\
\text { embryo transfer }\end{array}$ & (S) 499 & (S) 15037 & 7 \\
\hline
\end{tabular}


Table 1 Characteristics of 78 included cohort studies. S = Singleton pregnancy, M= Multiple pregnancy (Continued)

\begin{tabular}{|c|c|c|c|c|c|c|c|}
\hline $\begin{array}{l}\text { First author, } \\
\text { Publication } \\
\text { year, Country }\end{array}$ & $\begin{array}{l}\text { Type of } \\
\text { cohort }\end{array}$ & $\begin{array}{l}\text { Years of the } \\
\text { cohort }\end{array}$ & Matching factors & Comparison groups & $\begin{array}{l}\text { Pregnancies } \\
\text { conceived } \\
\text { by IVF/ICSI } \\
\text { (n) }\end{array}$ & $\begin{array}{l}\text { Spontaneous } \\
\text { pregnancies } \\
\text { (n) }\end{array}$ & $\begin{array}{l}\text { NOS } \\
\text { Score }\end{array}$ \\
\hline & Retrospective & & & & & & \\
\hline $\begin{array}{l}\text { Qin, 2017, China } \\
\text { [77] }\end{array}$ & $\begin{array}{l}\text { Hospital- } \\
\text { based } \\
\text { Prospective }\end{array}$ & 2013-2016 & No & IVF/ICSI & (S) 1260 & (S) 2480 & 6 \\
\hline $\begin{array}{l}\text { Raisanen, 2013, } \\
\text { Finland [78] }\end{array}$ & $\begin{array}{l}\text { Population- } \\
\text { based } \\
\text { Retrospective }\end{array}$ & 2006-2010 & No & IVF/ICSI & (S) 5647 & (S) 285357 & 7 \\
\hline $\begin{array}{l}\text { Reismullerova, } \\
\text { 2015, Slovakia } \\
\text { [79] }\end{array}$ & $\begin{array}{l}\text { Hospital- } \\
\text { based } \\
\text { Retrospective }\end{array}$ & N/A & No & IVF/ICSI & (S) 526 & (S) 15874 & 7 \\
\hline $\begin{array}{l}\text { Reubinoff, 1997, } \\
\text { Israel [80] }\end{array}$ & $\begin{array}{l}\text { Hospital- } \\
\text { based } \\
\text { Retrospective }\end{array}$ & 1983-1993 & $\begin{array}{l}\text { Maternal ethnic origin, age, } \\
\text { parity, location and date of } \\
\text { delivery }\end{array}$ & IVF/ICSI & (S) 260 & (S) 260 & 7 \\
\hline $\begin{array}{l}\text { Rizzo, 2016, Italy } \\
\text { [81] }\end{array}$ & $\begin{array}{l}\text { Hospital- } \\
\text { based } \\
\text { Prospective }\end{array}$ & 2007-2014 & Maternal age & $\begin{array}{l}\text { IVF/ICSI, Fresh } \\
\text { embryo transfer, } \\
\text { Frozen embryo } \\
\text { transfer }\end{array}$ & (S) 266 & (S) 266 & 9 \\
\hline $\begin{array}{l}\text { Rizzo, 2016, Italy } \\
{[2][82]}\end{array}$ & $\begin{array}{l}\text { Hospital- } \\
\text { based } \\
\text { Prospective }\end{array}$ & 2007-2014 & Maternal age & $\begin{array}{l}\text { IVF/ICSI, Oocyte } \\
\text { donation }\end{array}$ & (S) 109 & (S) 498 & 9 \\
\hline $\begin{array}{l}\text { Sazonova, 2012, } \\
\text { Sweden [83] }\end{array}$ & $\begin{array}{l}\text { Population- } \\
\text { based } \\
\text { Retrospective }\end{array}$ & 2002-2006 & No & $\begin{array}{l}\text { IVF/ICSI, Fresh } \\
\text { embryo transfer, } \\
\text { Frozen embryo } \\
\text { transfer }\end{array}$ & (S) 11292 & (S) 571914 & 7 \\
\hline $\begin{array}{l}\text { Shi, 2018, China } \\
\text { [84] }\end{array}$ & $\begin{array}{l}\text { Hospital- } \\
\text { based } \\
\text { Retrospective }\end{array}$ & 2013-2016 & No & IVF/ICSI & (M) 850 & (M) 250 & 5 \\
\hline $\begin{array}{l}\text { Shiloh, 2019, } \\
\text { Israel [85] }\end{array}$ & $\begin{array}{l}\text { Population- } \\
\text { based } \\
\text { Retrospective }\end{array}$ & 1991-2014 & No & IVF/ICSI & (S) 2603 & (S) 237863 & 6 \\
\hline $\begin{array}{l}\text { Silberstein, 2014, } \\
\text { Israel [86] }\end{array}$ & $\begin{array}{l}\text { Hospital- } \\
\text { based } \\
\text { Retrospective }\end{array}$ & 1988-2006 & No & IVF/ICSI & (S) 1294 & (S) 171513 & 6 \\
\hline $\begin{array}{l}\text { Stojnic, 2013, } \\
\text { Serbia [87] }\end{array}$ & $\begin{array}{l}\text { Hospital- } \\
\text { based } \\
\text { Retrospective }\end{array}$ & 2006-2010 & $\begin{array}{l}\text { Maternal age, parity, } \\
\text { education, time and place of } \\
\text { delivery, BMl }\end{array}$ & $\begin{array}{l}\text { IVF/ICSI, Fresh } \\
\text { embryo transfer }\end{array}$ & (S) 634 & (S)634 & 7 \\
\hline $\begin{array}{l}\text { Sun, 2009, } \\
\text { Canada [88] }\end{array}$ & $\begin{array}{l}\text { Population- } \\
\text { based } \\
\text { Retrospective }\end{array}$ & 2004-2007 & Maternal age, parity & IVF/ICSI & (S) 870 & (S) 3433 & 9 \\
\hline $\begin{array}{l}\text { Sun, 2016, China } \\
\text { [89] }\end{array}$ & $\begin{array}{l}\text { Hospital- } \\
\text { based } \\
\text { Retrospective }\end{array}$ & 2010-2014 & No & IVF/ICSI & (M) 411 & (M) 742 & 7 \\
\hline $\begin{array}{l}\text { Suzuki, 2010, } \\
\text { Japan [90] }\end{array}$ & $\begin{array}{l}\text { Hospital- } \\
\text { based } \\
\text { Retrospective }\end{array}$ & 2000-2007 & No & IVF/ICSI & (M) 64 & (M) 76 & 6 \\
\hline $\begin{array}{l}\text { Szymusik, 2012, } \\
\text { Poland [91] }\end{array}$ & $\begin{array}{l}\text { Hospital- } \\
\text { based } \\
\text { Retrospective }\end{array}$ & 2005-2009 & No & $\begin{array}{l}\text { IVF/ICSI, Fresh } \\
\text { embryo transfer }\end{array}$ & (M) 43 & $(M) 83$ & 4 \\
\hline $\begin{array}{l}\text { Szymusik, 2018, } \\
\text { Poland [92] }\end{array}$ & $\begin{array}{l}\text { Hospital- } \\
\text { based } \\
\text { Prospective }\end{array}$ & 2013-2016 & No & IVF/ICSI & (S) 183 & (S) 368 & 4 \\
\hline $\begin{array}{l}\text { Tan, 1992, UK } \\
\text { [93] }\end{array}$ & $\begin{array}{l}\text { Hospital- } \\
\text { based } \\
\text { Retrospective }\end{array}$ & 1978-1987 & Maternal age & IVF/ICSI & $\begin{array}{l}\text { (S) } 494 \\
\text { (M) } 125\end{array}$ & $\begin{array}{l}\text { (S) } 978 \\
\text { (M) } 21\end{array}$ & 6 \\
\hline Tandberg, 2015, & Population- & 1988-2009 & Parity & IVF/ICSI & (S) 12440 & (S) 1097084 & 8 \\
\hline
\end{tabular}


Table 1 Characteristics of 78 included cohort studies. S = Singleton pregnancy, M= Multiple pregnancy (Continued)

\begin{tabular}{|c|c|c|c|c|c|c|c|}
\hline $\begin{array}{l}\text { First author, } \\
\text { Publication } \\
\text { year, Country }\end{array}$ & $\begin{array}{l}\text { Type of } \\
\text { cohort }\end{array}$ & $\begin{array}{l}\text { Years of the } \\
\text { cohort }\end{array}$ & Matching factors & Comparison groups & $\begin{array}{l}\text { Pregnancies } \\
\text { conceived } \\
\text { by IVF/ICSI } \\
\text { (n) }\end{array}$ & $\begin{array}{l}\text { Spontaneous } \\
\text { pregnancies } \\
\text { (n) }\end{array}$ & $\begin{array}{l}\text { NOS } \\
\text { Score }\end{array}$ \\
\hline Norway [94] & $\begin{array}{l}\text { based } \\
\text { Retrospective }\end{array}$ & & & & & & \\
\hline $\begin{array}{l}\text { Tomic, 2011, } \\
\text { Croatia [95] }\end{array}$ & $\begin{array}{l}\text { Hospital- } \\
\text { based } \\
\text { Retrospective }\end{array}$ & 2006-2009 & $\begin{array}{l}\text { Ethnicity, age, gravidity, } \\
\text { smoking habits, BMl, weight } \\
\text { gain in pregnancy, site and } \\
\text { time of delivery }\end{array}$ & $\begin{array}{l}\text { IVF/ICSI, Fresh } \\
\text { embryo transfer }\end{array}$ & (S) 283 & (S) 283 & 7 \\
\hline $\begin{array}{l}\text { Valenzuela- } \\
\text { Alcaraz, 2013, } \\
\text { Spain [96] }\end{array}$ & $\begin{array}{l}\text { Hospital- } \\
\text { based } \\
\text { Prospective }\end{array}$ & $\mathrm{N} / \mathrm{A}$ & Age & IVF/ICSI & (S) 100 & (S) 100 & 6 \\
\hline $\begin{array}{l}\text { Valenzuela- } \\
\text { Alcaraz, 2018, } \\
\text { Spain [97] }\end{array}$ & $\begin{array}{l}\text { Hospital- } \\
\text { based } \\
\text { Prospective }\end{array}$ & 2014-2016 & No & IVF/ICSI & (M) 50 & (M) 50 & 7 \\
\hline $\begin{array}{l}\text { Vasario, 2012, } \\
\text { Italy [98] }\end{array}$ & $\begin{array}{l}\text { Hospital- } \\
\text { based } \\
\text { Prospective }\end{array}$ & 2004-2008 & No & IVF/ICSI & (M) 84 & (M) 139 & 6 \\
\hline $\begin{array}{l}\text { Von Versen- } \\
\text { Hoynck, 2019, US } \\
\text { [99] }\end{array}$ & $\begin{array}{l}\text { Hospital- } \\
\text { based } \\
\text { Prospective }\end{array}$ & $2011-2017$ & No & $\begin{array}{l}\text { IVF/ICSI, Fresh } \\
\text { embryo transfer, } \\
\text { Frozen embryo } \\
\text { transfer }\end{array}$ & (S) 367 & (S) 143 & 5 \\
\hline $\begin{array}{l}\text { Watanabe, 2014, } \\
\text { Japan [100] }\end{array}$ & $\begin{array}{l}\text { Hospital- } \\
\text { based } \\
\text { Retrospective }\end{array}$ & 2009-2011 & $\begin{array}{l}\text { Closest propensity score } \\
\text { (accounting for } 27 \text { maternal } \\
\text { and paternal variables) }\end{array}$ & IVF/ICSI & (S) 474 & (S) 474 & 9 \\
\hline $\begin{array}{l}\text { Wennberg, 2016, } \\
\text { Sweden, } \\
\text { Denmark, } \\
\text { Finland, Norway } \\
{[101]}\end{array}$ & $\begin{array}{l}\text { Population- } \\
\text { based } \\
\text { Retrospective }\end{array}$ & 1982-2007 & $\begin{array}{l}\text { Parity, year and month of } \\
\text { birth }\end{array}$ & $\begin{array}{l}\text { IVF/ICSI, Fresh } \\
\text { embryo transfer }\end{array}$ & (S) 39919 & (S) 260166 & 7 \\
\hline $\begin{array}{l}\text { Wu, 2010, China } \\
{[102]}\end{array}$ & $\begin{array}{l}\text { Hospital- } \\
\text { based } \\
\text { Retrospective }\end{array}$ & $2006-2008$ & No & IVF/ICSI & (M) 204 & (M) 255 & 4 \\
\hline $\begin{array}{l}\text { Xu, 2005, China } \\
{[103]}\end{array}$ & $\begin{array}{l}\text { Hospital- } \\
\text { based } \\
\text { Retrospective }\end{array}$ & $2001-2003$ & No & IVF/ICSI & (M) 41 & (M) 44 & 4 \\
\hline $\begin{array}{l}\text { Yang, 2011, } \\
\text { Korea [104] }\end{array}$ & $\begin{array}{l}\text { Hospital- } \\
\text { based } \\
\text { Retrospective }\end{array}$ & 1995-2008 & No & IVF/ICSI & (M) 67 & (M) 143 & 7 \\
\hline $\begin{array}{l}\text { Yang, 2014, } \\
\text { China [105] }\end{array}$ & $\begin{array}{l}\text { Population- } \\
\text { based } \\
\text { Retrospective }\end{array}$ & 2011 & No & IVF/ICSI & $\begin{array}{l}\text { (S) } 825 \\
\text { (M) } 314\end{array}$ & $\begin{array}{l}\text { (S) } 109971 \\
\text { (M) } 1473\end{array}$ & 6 \\
\hline $\begin{array}{l}\text { Zadori, 2003, } \\
\text { Hungary [106] }\end{array}$ & $\begin{array}{l}\text { Hospital- } \\
\text { based } \\
\text { Retrospective }\end{array}$ & 1995-2002 & $\begin{array}{l}\text { Maternal age, parity, gravidity, } \\
\text { previous obstetrics outcomes }\end{array}$ & IVF/ICSI & $\begin{array}{l}\text { (S) } 185 \\
\text { (M) } 36\end{array}$ & $\begin{array}{l}\text { (S) } 185 \\
\text { (M) } 36\end{array}$ & 6 \\
\hline $\begin{array}{l}\text { Zhang, 2015, } \\
\text { China [107] }\end{array}$ & $\begin{array}{l}\text { Hospital- } \\
\text { based } \\
\text { Retrospective }\end{array}$ & 2010-2014 & No & IVF/ICSI & (M) 53 & (M) 128 & 6 \\
\hline $\begin{array}{l}\text { Zhu, 2016, China } \\
\text { [108] }\end{array}$ & $\begin{array}{l}\text { Hospital- } \\
\text { based } \\
\text { Retrospective }\end{array}$ & 2006-2014 & Maternal age, birth year & IVF/ICSI & $\begin{array}{l}\text { (S) } 1659 \\
\text { (M) } 982\end{array}$ & $\begin{array}{l}\text { (S) } 5193 \\
\text { (M) } 89\end{array}$ & 9 \\
\hline
\end{tabular}




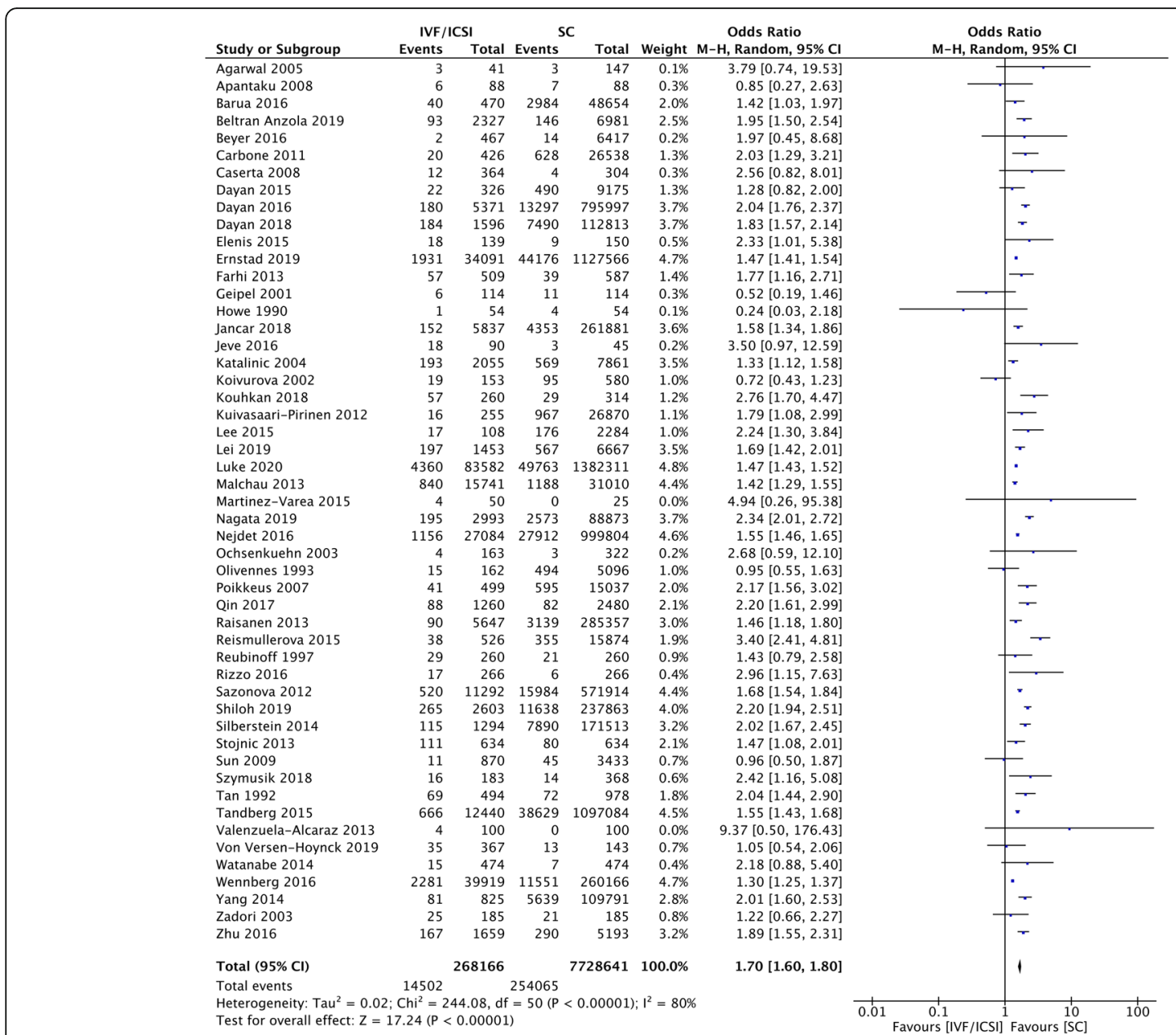

Fig. 2 IVF/ICSI Singleton Pregnancies Meta-analysis. Forest plot comparing hypertensive disorders of pregnancy in IVF/ICSI singleton pregnancies in comparison to spontaneous pregnancies

case of preeclampsia, only two studies were eligible with no significant difference between the two groups (OR 0.98, 95\% CI 0.38-2.51; $\mathrm{I}^{2}=72 \%$ ) (Additional file 5). Both pooled analyses showed high heterogeneity.

\section{IVF/ICSI multiple pregnancies}

Forty-one studies assessed HDP in multiple pregnancies. Higher odds were observed in the IVF/ICSI group than the SC group, with an OR of 1.34 (95\% CI 1.20-1.50) with high heterogeneity $\left(\mathrm{I}^{2}=76 \%\right.$ ) (Fig. 4). The number of studies that were rated as high, moderate, and low quality by NOS scores were 8,28 , and 5 respectively.

Seventeen studies assessed preeclampsia as the outcome of interest. The odds of preeclampsia were higher in pregnancies resulting from IVF/ICSI than SC (OR $1.24,95 \%$ CI 1.08-1.43) with low heterogeneity $\left(\mathrm{I}^{2}=\right.$ 32\%) (Additional file 5). All studies either had moderate or high quality.

For IVF pregnancies, four and two studies looked at HDP and preeclampsia respectively. Both showed slightly increased odds in the ART groups in comparison to $\mathrm{SC}$; however, the differences were insignificant (HDP: OR 1.13, 95\% CI 0.98-1.29; $\mathrm{I}^{2}=59 \%$. Preeclampsia: OR 1.04, 95\% CI $0.93-1.16 ; \quad I^{2}=0 \%$ ) (Fig. 5, Additional file 5).

For ICSI multiple pregnancies, 10 studies were eligible for the analysis of HDP. Overall, no increase in the risk of HDP was observed in the exposure group (OR 1.11, 95\% CI 0.91-1.36; $\mathrm{I}^{2}=77 \%$ ) (Fig. 3). Five studies reported data on preeclampsia in multiple pregnancies after ICSI. The odds of preeclampsia were slightly higher in pregnancies resulting from ICSI than SC (OR 1.11, 95\% CI 1.00-1.24) in the pooled analysis with no heterogeneity $\left(\mathrm{I}^{2}=0 \%\right)$ (Additional file 5$)$.

\section{Fresh and frozen embryo transfer}

Sixteen studies reported on the relationship between fresh ET and HDP. The pooled result showed that, when compared to SC, fresh ET is associated with increased odds of HDP with an OR of 1.43 (95\% CI 


\section{a) IVF singleton pregnancies}

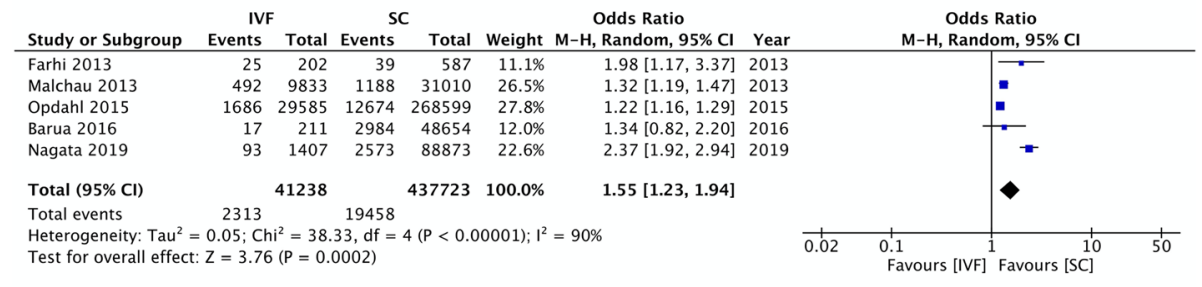

\section{b) ICSI singleton pregnancies}

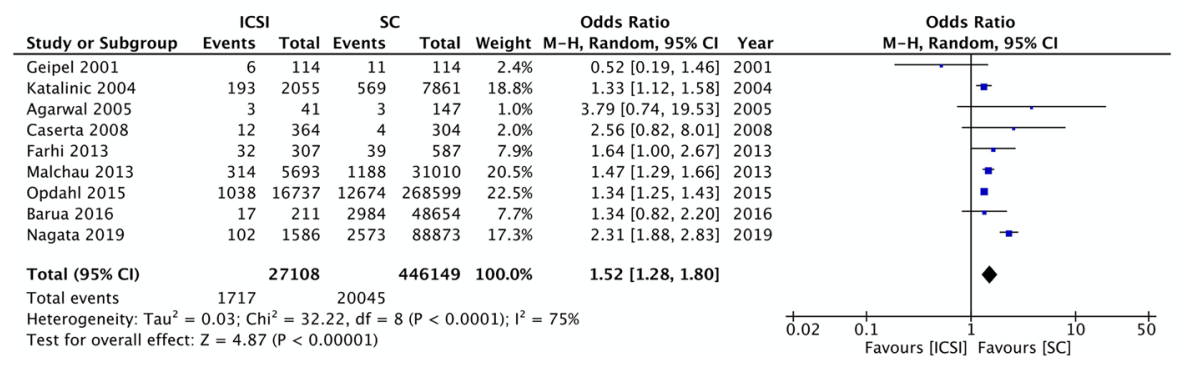

Fig. 3 IVF and ICSI Singleton Pregnancies Meta-analysis. Forest plot comparing hypertensive disorders of pregnancy in a) IVF singleton pregnancies and $\mathbf{b}$ ) ICSI singleton pregnancies in comparison to spontaneous pregnancies

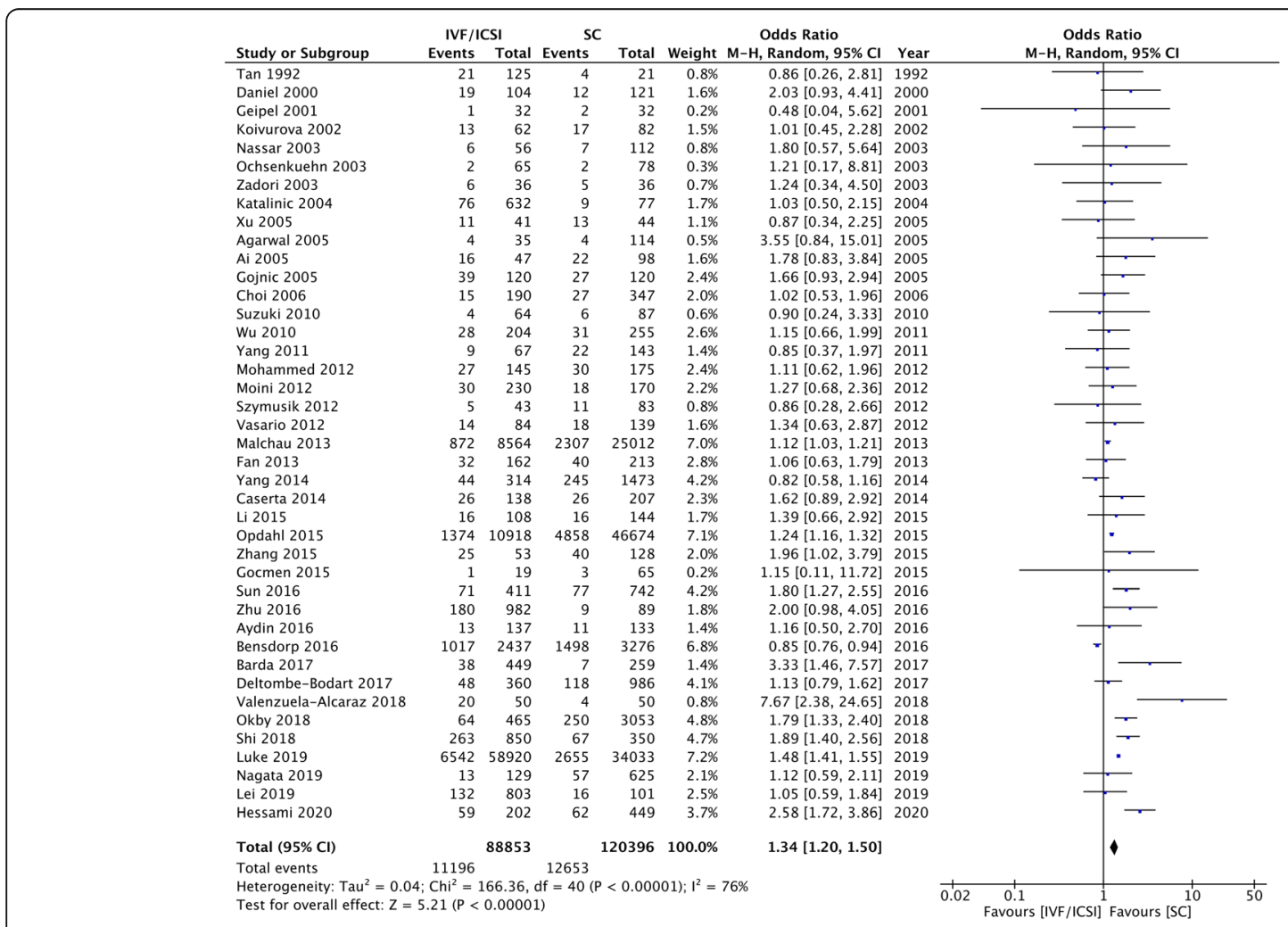

Fig. 4 IVF/ICSI Multiple Pregnancies Meta-analysis. Forest plot comparing hypertensive disorders of pregnancy in IVF/ICSI multiple pregnancies in comparison to spontaneous pregnancies 


\section{a) IVF multiple pregnancies}

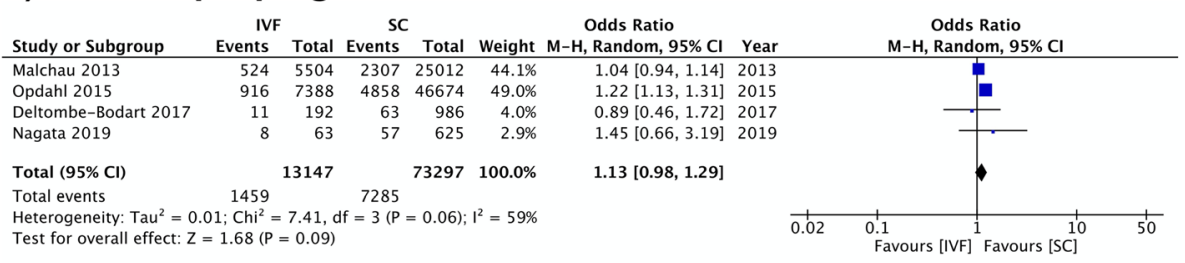

\section{b) ICSI multiple pregnancies}

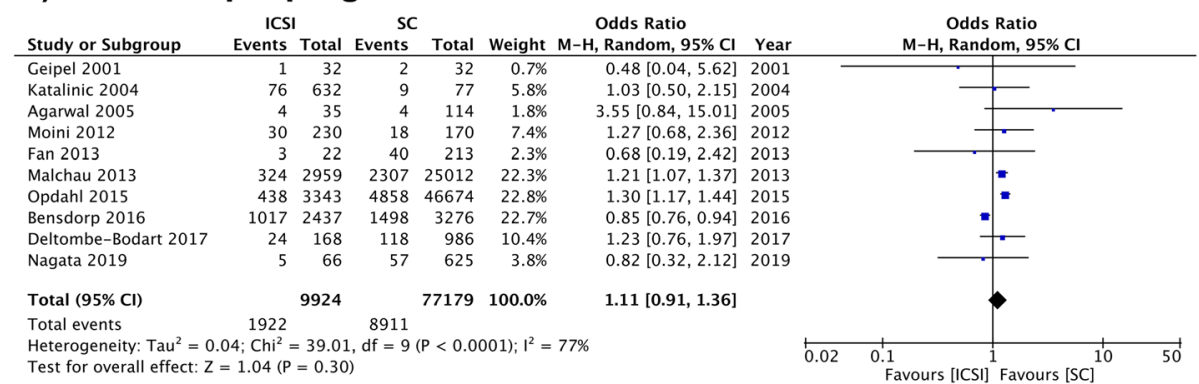

Fig. 5 IVF and ICSI Multiple Pregnancies Meta-analysis Forest plot comparing hypertensive disorders of pregnancy in a) IVF multiple pregnancies and b) ICSI multiple pregnancies in comparison to spontaneous pregnancies

\section{a) Fresh embryo transfer}

\begin{tabular}{|c|c|c|c|c|c|c|c|c|c|c|c|}
\hline \multirow[b]{2}{*}{ Study or Subgroup } & \multicolumn{2}{|c|}{ IVF/ICSI } & \multicolumn{2}{|c|}{ sc } & \multicolumn{3}{|c|}{ Odds Ratio } & \multirow{2}{*}{\multicolumn{4}{|c|}{$\begin{array}{c}\text { Odds Ratio } \\
\text { M-H, Random, } 95 \% \mathrm{Cl}\end{array}$}} \\
\hline & Events & Total & Events & Total & Weight & $\mathrm{M}-\mathrm{H}, \mathrm{R}$ andom, $95 \% \mathrm{Cl}$ & Year & & & & \\
\hline Koivurova 2002 & 19 & 153 & 95 & 580 & $1.5 \%$ & $0.72[0.43,1.23]$ & 2002 & & & & \\
\hline Katalinic 2004 & 193 & 2055 & 569 & 7861 & $8.2 \%$ & $1.33[1.12,1.58]$ & 2004 & & & $\mp$ & \\
\hline Poikkeus 2007 & 41 & 499 & 595 & 15037 & $3.5 \%$ & $2.17[1.56,3.02]$ & 2007 & & & - & \\
\hline Tomic 2011 & 4 & 283 & 2 & 283 & $0.2 \%$ & $2.01[0.37,11.09]$ & 2011 & & & & \\
\hline Sazonova 2012 & 395 & 8944 & 15984 & 571914 & $12.3 \%$ & $1.61[1.45,1.78]$ & 2012 & & & $=$ & \\
\hline Stojnic 2013 & 111 & 634 & 80 & 634 & $3.8 \%$ & $1.47[1.08,2.01]$ & 2013 & & & - & \\
\hline Rizzo 2016 & 11 & 139 & 6 & 266 & $0.4 \%$ & $3.72[1.35,10.30]$ & 2016 & & & & \\
\hline Wennberg 2016 & 2281 & 39919 & 11551 & 260166 & $15.7 \%$ & $1.30[1.25,1.37]$ & 2016 & & & - & \\
\hline Beyer 2016 & 1 & 188 & 14 & 6417 & $0.1 \%$ & $2.45[0.32,18.70]$ & 2016 & & & & \\
\hline Korosec 2016 & 43 & 916 & 147 & 2748 & $3.2 \%$ & $0.87[0.62,1.23]$ & 2016 & & & & \\
\hline Nejdet 2016 & 825 & 20290 & 27912 & 999804 & $14.3 \%$ & $1.48[1.38,1.58]$ & 2016 & & & - & \\
\hline Szymusik 2018 & 16 & 183 & 14 & 368 & $0.8 \%$ & $2.42[1.16,5.08]$ & 2018 & & & & \\
\hline Beltran Anzola 2019 & 81 & 1961 & 128 & 5883 & $4.4 \%$ & $1.94[1.46,2.57]$ & 2019 & & & - & \\
\hline Ernstad 2019 & 1268 & 24365 & 44176 & 1127566 & $15.1 \%$ & $1.35[1.27,1.43]$ & 2019 & & & . & \\
\hline Von Versen-Hoynck 2019 & 8 & 146 & 10 & 143 & $0.5 \%$ & $0.77[0.30,2.01]$ & 2019 & & & & \\
\hline Luke 2020 & 3156 & 62192 & 49763 & 1382311 & $16.1 \%$ & $1.43[1.38,1.49]$ & 2020 & & & - & \\
\hline Total $(95 \% \mathrm{Cl})$ & & 162867 & & 4381981 & $100.0 \%$ & $1.43[1.33,1.53]$ & & & & 1 & \\
\hline Total events & 8453 & & 151046 & & & & & & & & \\
\hline $\begin{array}{l}\text { Heterogeneity: } \text { Tau }^{2}=0.01 \\
\text { Test for overall effect: } Z=\end{array}$ & $\begin{array}{l}\mathrm{Chi}^{2}=54 \\
0.17(\mathrm{P}<\end{array}$ & $\begin{array}{l}7.41, \mathrm{df}= \\
0.00001\end{array}$ & $=15(P<$ & 0000 & 72 & & & 0.02 & $\begin{array}{c}0.1 \\
\text { Favours }\end{array}$ & 1 Favours [SC] & 50 \\
\hline
\end{tabular}

\section{b) Frozen embryo transfer}

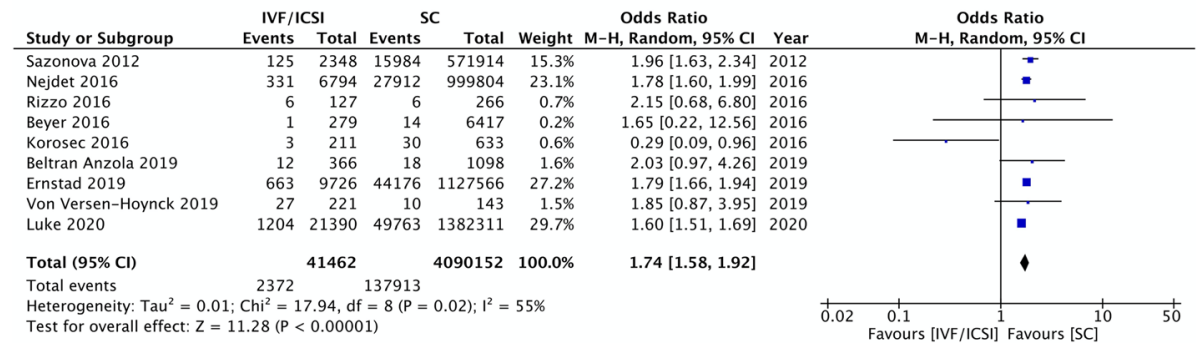

Fig. 6 Fresh and Frozen Embryo Transfer Meta-analysis. Forest plot comparing hypertensive disorders of pregnancy in singleton pregnancies resulting from a) fresh embryo transfer or $\mathbf{b})$ frozen embryo transfer in comparison to spontaneous pregnancies 


\section{a) Oocyte donation singleton pregnancies}

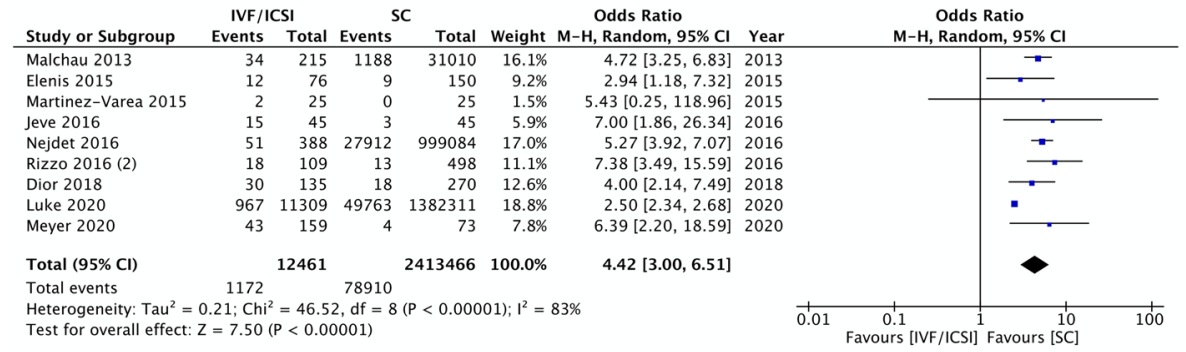

\section{b) Oocyte donation multiple pregnancies}

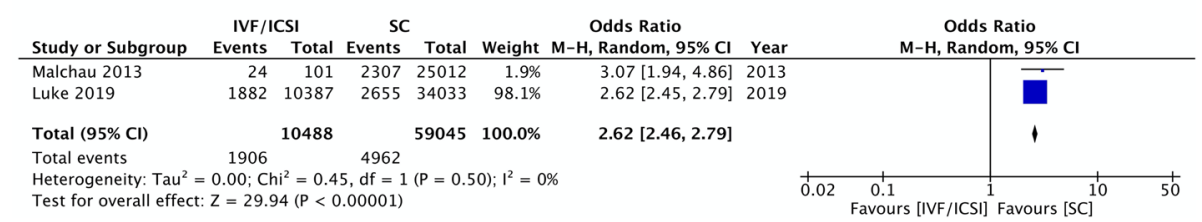

Fig. 7 Oocyte Donation Meta-analysis. Forest plot comparing hypertensive disorders of pregnancy in a) singleton pregnancies or $\mathbf{b}$ ) multiple pregnancies resulting from oocyte donation in comparison to spontaneous pregnancies

$1.33-1.53 ; \mathrm{I}^{2}=72 \%$ ) (Fig. 6). A similar finding was also found in the pooled result of eight studies using preeclampsia as the outcome of interest (OR 1.48, 95\% CI $1.37-1.60)$ with low heterogeneity $\left(I^{2}=39 \%\right)$ (Additional file 5).
FET was also associated with higher odds of HDP and preeclampsia compared to SC. Nine studies were included resulting in a pooled OR of 1.74 (95\% CI $1.58-1.92 ; \mathrm{I}^{2}=55 \%$ ) for HDP (Fig. 6). Comparably, in five studies that studied preeclampsia, the OR was

Table 2 Summary of results by type of ART and outcome of interest

\begin{tabular}{|c|c|c|c|c|c|c|c|}
\hline Experimental & Number of studies & ART study size (n) & SC study size (n) & OR; 95\% Cl & $I^{2}(\%)$ & $P$ value & NNH (n) \\
\hline \multicolumn{8}{|l|}{ Hypertensive Disorders of Pregnancy } \\
\hline IVF/ICSI singleton & 51 & 268,166 & $7,728,641$ & $1.70(1.60-1.80)$ & 80 & $<0.01^{\mathrm{a}}$ & 47.2 \\
\hline IVF singleton & 5 & 41,238 & 437,723 & $1.55(1.23-1.94)$ & 90 & $<0.01^{\mathrm{a}}$ & 85.9 \\
\hline ICSI singleton & 9 & 27,108 & 446,149 & $1.52(1.28-1.80)$ & 75 & $<0.01^{\mathrm{a}}$ & 54.3 \\
\hline IVF/ICSI multiple & 41 & 88,853 & 120,396 & $1.34(1.20-1.50)$ & 76 & $<0.01^{\mathrm{a}}$ & 47.8 \\
\hline IVF multiple & 4 & 13,147 & 73,297 & $1.13(0.98-1.29)$ & 59 & 0.09 & 86.3 \\
\hline ICSI multiple & 10 & 9924 & 77,179 & $1.11(0.91-1.36)$ & 77 & 0.30 & 12.8 \\
\hline Fresh embryo transfer singleton & 16 & 162,867 & $4,381,981$ & $1.43(1.33-1.53)$ & 72 & $<0.01^{\mathrm{a}}$ & 57.4 \\
\hline Frozen embryo transfer singleton & 9 & 41,462 & $4,090,152$ & $1.74(1.58-1.92)$ & 55 & $<0.01^{\mathrm{a}}$ & 52.6 \\
\hline Oocyte donation singleton & 9 & 12,461 & $2,413,466$ & $4.42(3.00-6.51)$ & 83 & $<0.01^{\mathrm{a}}$ & 16.3 \\
\hline Oocyte donation multiple & 2 & 10,488 & 59,045 & $2.62(2.46-2.79)$ & 0 & $<0.01^{\mathrm{a}}$ & 10.2 \\
\hline \multicolumn{8}{|l|}{ Preeclampsia } \\
\hline IVF/ICSI singleton & 28 & 118,787 & $4,509,905$ & $1.59(1.46-1.74)$ & 70 & $<0.01^{\mathrm{a}}$ & 81.0 \\
\hline ICSI singleton & 2 & 5807 & 31,124 & $0.98(0.38-2.51)$ & 72 & 0.97 & 105.2 \\
\hline IVF/ICSI multiple & 17 & 14,842 & 34,337 & $1.24(1.08-1.43)$ & 32 & $<0.01^{\mathrm{a}}$ & 91.7 \\
\hline IVF multiple & 2 & 5696 & 25,998 & $1.04(0.93-1.16)$ & 0 & 0.47 & 364.0 \\
\hline ICSI multiple & 5 & 5618 & 29,519 & $1.11(1.00-1.24)$ & 0 & $0.05^{\mathrm{a}}$ & 52.1 \\
\hline Fresh embryo transfer singleton & 8 & 55,300 & $2,715,647$ & $1.48(1.37-1.60)$ & 39 & $<0.01^{\mathrm{a}}$ & 85.5 \\
\hline Frozen embryo transfer singleton & 5 & 19,216 & $2,699,693$ & $1.82(1.71-1.95)$ & 0 & $<0.01^{\mathrm{a}}$ & 45.6 \\
\hline Oocyte donation singleton & 7 & 1107 & $1,031,110$ & $5.20(4.02-6.73)$ & 9 & $<0.01^{\mathrm{a}}$ & 10.1 \\
\hline
\end{tabular}

${ }^{a}=$ Statistically significant. $N N H$ Numbers needed to harm 
1.82 (95\% CI 1.71-1.95) with no heterogeneity between included studies $\left(\mathrm{I}^{2}=0 \%\right)$ (Additional file 5 ).

\section{Oocyte donation}

Pregnancies resulting from OD were found to have the highest risk of hypertensive complications of all analyses conducted for the study. For HDP, nine studies resulted in a pooled OR of 4.42 (95\% CI 3.00-6.51; I ${ }^{2}=83 \%$ ) (Fig. 7). Similar findings were observed in multiple pregnancies, with an OR of 2.62 (95\% CI 2.46-2.79) with no heterogeneity $\left(\mathrm{I}^{2}=0 \%\right)$. However, only two studies were eligible (Fig. 7).

Seven studies studied preeclampsia as the outcome of interest. The resulting OR was 5.20 (95\% CI 4.02-6.73) with low heterogeneity $\left(\mathrm{I}^{2}=9 \%\right)$ (Additional file 5 ). All included studies had a moderate or high quality according to their NOS scores.

Overall, all IVF/ICSI groups were associated with increased odds of HDP in comparison to SC; however, the differences were insignificant when selected IVF and ICSI multiple pregnancies were analyzed separately. Similarly, all study groups except ICSI singleton pregnancies were associated with higher odds of preeclampsia. The difference between IVF multiple pregnancies and SC was small and insignificant. All findings described above were summarized in Table 2 .

\section{Numbers needed to harm}

The numbers needed to harm for each IVF/ICSI subgroups were shown in Table 2. While most interventions had an NNH of 40 to 100 , OD pregnancies had particularly low NNH. Sixteen patients who achieved singleton pregnancies through OD were needed to have one case of HDP; similarly, only ten multiple pregnancy patients through OD were needed to have one case of HDP.

\section{Sensitivity analysis and publication bias}

Sensitivity analyses were performed to identify individual studies with large influences on the overall risk estimates. Exclusion of any study did not yield significantly different OR, with the exception of one study by Malchau et al. for the analysis of preeclampsia in IVF multiple pregnancies (Additional file 6). Funnel plots of meta-analyses involving more than 10 studies did not reveal any publication bias (Additional file 5).

\section{Discussion}

\section{Principle findings}

IVF/ICSI pregnancies, when compared to SC, carried higher odds of HDP and preeclampsia regardless of their plurality. While both fresh ET and FET were found to have odds of hypertensive complications, FET was associated with higher odds in comparison fresh ET.
Pregnancies resulting from OD had the highest odds of HDP and preeclampsia out of all the groups analyzed.

Analyses on IVF and ICSI pregnancies specifically yielded mixed results and were limited by a relatively small number of studies. Although IVF pregnancies had higher incidences of HDP in comparison to SC, the difference was not statistically significant for multiple pregnancies. While ICSI singleton pregnancies resulted in higher odds of HDP compared to SC, the odds of preeclampsia were similar in both groups. In multiple pregnancy, although ICSI was associated with increased odds of preeclampsia, the difference with SC was small.

\section{Comparison with existing literature}

Our results of higher odds of HDP and preeclampsia in singleton pregnancies following IVF/ICSI were in accordance with other previous meta-analyses $[7,10$, 13, 14]. The measures of association for HDP in IVF/ ICSI pregnancies (OR 1.70; 95\% CI 1.60-1.80) was comparable to meta-analyses by Panday et al. (RR 1.49; 95\% CI 1.39-1.59) and Qin et al. (RR 1.30; 95\% CI 1.04-1.62) with a significant increase in statistical power due to a high number of studies included (51 studies) [7, 14]. Although the most recent metaanalysis by Thomopoulos et al. did not report data for IVF/ICSI pregnancies, the estimated relative risk for HDP in IVF pregnancies (RR 1.45; 95\% CI 1.261.68) was similar to our study result (RR 1.55 ; $95 \%$ CI 1.23-1.94) [10]. This comprehensive systemic review included some cohort studies with mixed gestational orders, which was a strict exclusion criterion for our meta-analysis to minimize confounding bias. Our study results could not be directly compared to those by Almasi-Hashiani et al. as the study also included fertility treatments other than IVF/ICSI in the exposure group [13].

Multiple pregnancy following IVF/ICSI was also found to be at higher odds for preeclampsia and HDP, although the differences were smaller. This was likely because HDP and preeclampsia are known have a higher prevalence in multiple pregnancies than singleton pregnancies, resulting in higher risks of hypertensive complications in both SC and IVF/ICSI groups [68]. The metaanalysis by Qin et al. published in 2015 comprehensively studied the risk of HDP in IVF/ICSI multiple pregnancy. However, the study included other fertility treatments, such as ovulation induction and intrauterine insemination, in the control group, potentially underestimating the risk of IVF/ICSI on the outcome of interest. This might explain the slightly lower relative risk (RR 1.13; 95\% CI 1.02-1.26) in comparison to our findings (OR 1.34; 95\% CI 1.20-1.50) [9].

When studying ICSI pregnancies separately, our study found varying results for preeclampsia and HDP, and 
the findings were consistent with those reported by Thomopoulos and colleagues. While the meta-analysis by Thomopoulos et al. was limited by the small number of available studies, our sub-analysis included more recent studies and yielded similar findings [10]. Further research on ICSI pregnancies, as well as their indications for the procedure, such as male infertility, should be conducted to identify potential contributing factors.

Although the mechanism by which IVF/ICSI increases the risk of preeclampsia remains unknown, there exists a great body of literature studying potential causes. First, baseline maternal characteristics such as advanced maternal age, obesity, and medical comorbidities vary between the exposure and control groups and have been shown to be associated with preeclampsia $[38,94]$. The underlying infertility diagnosis may also lead to varying maternal and perinatal outcomes. A large-scale cohort study by Stern and colleagues found that women with infertility due to tubal factors and ovulation disorders may be at a particularly high risk of HDP in comparison to their respective SC groups with the same diagnoses [8]. Patients with endometriosis may carry a higher risk of HDP than the general population, although the risk is attenuated when specifically studying women with ART [109]. Together, these findings suggest that an individual's risk of HDP must be evaluated based on the mode of conception, along with other patient factors and comorbidities.

The presence of significant differences in the outcomes between fresh ET, FET, and OD pregnancies highlighted the potential role of the procedure on the development of hypertensive complications. In recent years, the use of cryopreservation has expanded widely from women with medical indications (e.g. with medical conditions or treatments that impairs fertility) to social embryo and oocyte freezing, including women who prefer to defer childbearing and transgender people as a part of their medical transition process [110]. There is a growing interest in the "freeze-all" strategy as a result of its decreased incidence of ovarian hyperstimulation syndrome without compromising live birth rates [110]. While FET carries many unique advantages, it is still important to understand the associated perinatal outcomes with the procedure. Our review of all available literature showed that both fresh ET and FET were associated with increased risks of preeclampsia and HDP in comparison to SC; furthermore, the observed differences were greater in FET pregnancies than fresh ET pregnancies, which was consistent with earlier evidence $[17,111,112]$. It is worth noting that most past literature used fresh ET as a control group instead, making it difficult to directly compare the results between studies.
The observed difference between fresh ET and FET may be explained by the absence of a corpus luteum (CL), as suggested by five recently published cohort studies [44, 99, 113-115]. SC typically develop under the presence of one $\mathrm{CL}$, while the number of CL for IVF pregnancies varies depending on the type of procedure. Fresh IVF cycles typically involve more than one CL, whereas frozen IVF cycles and OD are usually performed under programmed cycles with exogenous hormones in the absence of a CL $[113,115]$.

von Versen-Hoynck and colleagues performed a prospective cohort study showing that women who conceived without a CL had a higher risk of preeclampsia than women with one or more CL. In the same paper, further analysis also demonstrated that programmed FET pregnancies were associated with higher incidences of preeclampsia than natural FET pregnancies, which had a comparable risk as fresh IVF pregnancies [99]. This finding was again supported by Luke and colleagues [115]. These studies proposed that performing FET during a natural cycle or with supplementation of missing hormones, such as relaxin, may potentially reduce the risk of preeclampsia and HDP. However, this theory alone could not explain the increased risk in fresh ET in comparison to SC, suggesting that there may be other components of the procedure or unaccounted confounders that increased the risk of hypertensive complications. As fresh ET pregnancies were found to be associated with other perinatal complications such as low birth weight and small for gestational age, it is important to balance the risks and benefits with each patient's health status when considering treatment options [116].

OD is becoming a common standard practice for patients with reproductive disorders, diminished ovarian reserve, or advanced maternal age due to its relatively high success rate and comparable live delivery rates in comparison to autologous IVF pregnancies $[117,118]$. In our study, women who achieved singleton pregnancies from donated oocytes carried fourto five-fold odds of preeclampsia and HDP in comparison to women who achieved pregnancy through SC. Multiple pregnancies from OD also had higher odds, although the differences with spontaneous multiple pregnancies were smaller.

This finding was consistent with other previous systematic reviews and meta-analyses that used SC as the control group $[5,119,120]$. Pecks et al. reported the OR for HDP in OD pregnancies in comparison to SC to be 6.60 (95\% CI 4.55-9.57). However, some included studies did not adjust for plurality, potentially leading to an overestimation due to the known risk of multiple pregnancy. Similarly, Masoudian et al. calculated an odds 
ratio of 4.34 (95\% CI 3.10-6.06) with studies that included both singleton and multiple pregnancies. Storgaard et al. reported an odds ratio of 2.45 (95\% CI 1.531.93) and an odds ratio of 2.95 (95\% CI 2.29-3.76) for HDP and preeclampsia, respectively. Since the most recent literature, five other cohort studies were published and included in our analyses $[42,43,53,63,66]$.

In addition to the potential role of CL on maternal circulation, the increased risk of preeclampsia and HDP observed in women who conceived via OD has also been suggested to be a result of a heighten immunologic response between the mother and the allogenic oocyte [121]. This was formed on the basis that normal placentation requires the development of immunologic tolerance of the mother and the fetus; studies reporting an increased risk of preeclampsia in primiparous women and after a change in paternity in multiparous women further support this immunologic theory [121, 122]. Lashley and colleagues found that among successful and uncomplicated OD pregnancies, there was a higher level of human leukocyte antigen (HLA) matching between mother and fetus in than expected by chance, suggesting the role of HLA gene in the development of preeclampsia [123]. However, it is still important to consider other patient factors such as advanced maternal age, which is very common in this patient population and may also play a role to the increased risk of hypertensive disorders.

\section{Strengths and limitations}

Our findings were generally consistent with previous literature; however, this study also carries many distinctive strengths. This is the most up-to-date, largescale meta-analysis (including 85 studies and 8.5 million pregnancies) studying the association between IVF/ICSI pregnancies and HDP. Strict inclusion and exclusion criteria were employed to focus solely on IVF/ICSI pregnancies and SC (without any fertility treatment) to provide findings that are unique to this ART procedure. Recognizing the inherent risk of HDP in multiple pregnancies, all analyses stratified patients by plurality. Two outcomes, preeclampsia and HDP, were reported separately due to their differences in risk and prognosis. Furthermore, 89.4\% of all included studies were of moderate to high quality (Table 1, Additional file 4). Finally, all publications in English, Chinese, Portuguese, and French were screened and reviewed to minimize language bias.

However, there were also some limitations. IVFspecific analyses were limited by the low number of studies that explicitly excluded ICSI pregnancies. The diagnostic criteria of preeclampsia differ depending on the study period and geographical location [75]. The lack of definition for the outcomes of interest among the cohorts (43\% of all included studies) also made it difficult to create a uniform definition for the meta-analysis. This issue was solved by considering the following criteria: if a study used preeclampsia as an outcome without specifying the definition, it was included in analyses for preeclampsia. If a study used terminologies such as "pregnancy-induced hypertension", "gestational hypertension", or "hypertensive disorder", it was only included in analyses for HDP. A high heterogeneity was reported in many pooled analyses, likely due to differences in study populations and geographical areas. Finally, uncontrolled confounders remained to be a concern due to the nature of the study design and could also influence heterogeneity. Some of the included studies did employ a matching method when selecting control subjects, while other studies accounted for potential confounders such as age, parity, medical comorbidities, year of birth, socioeconomic status, ethnic origin, location, and cause of infertility.

\section{Conclusions and implications}

There has been an increasing amount of literature studying the relationship between ART and pregnancy and perinatal outcomes over the past decade, but there is also a lack of clinical practice guidelines for women who conceived through ART [124]. Results of our meta-analyses confirmed that IVF/ICSI pregnancies were at high odds of preeclampsia and HDP than $\mathrm{SC}$, irrespective of the plurality. In particular, the odds in FET and OD pregnancies were high. Further population-based research studying different IVF treatment protocols should be considered. Health care providers should be aware of these risks and develop specific care plans and interventions for pregnancies conceived by IVF/ICSI to decrease the incidence of hypertensive complications and subsequently the risks of maternal morbidity and mortality. For example, a systematic review of randomized controlled trials by Henderson et al. suggested that daily low-dose aspirin starting after the first trimester might reduce the risk of preeclampsia [125]. The relationship between preeclampsia and ICSI singleton pregnancies remains unclear due to insufficient literature that studies this population. Given that the use of ICSI is gaining popularity over time, more research studying the pregnancy outcomes after ICSI is warranted.

\section{Abbreviations}

ART: Assisted reproductive technologies; IVF: In vitro fertilization; ICSI: Intracytoplasmic sperm injection; Fresh ET: Fresh embryo transfer; FET: Frozen embryo transfer; OD: Oocyte donation; HDP: Hypertensive disorders of pregnancy; SC: Spontaneous conception; PRISMA: Preferred Reporting Items for Systematic Reviews and Meta-analyses; NOS: Newcastle Ottawa Scale; OR: Odds ratio; CL: Corpus luteum; HLA: Human leukocyte antigen 


\section{Supplementary Information}

The online version contains supplementary material available at https://doi. org/10.1186/s12884-021-03938-8.

Additional file 1. Preferred Reporting Items for Systematic Reviews and Meta-Analyses (PRISMA) checklist. A list of 27 items required under the PRISMA statement with their respective locations.

Additional file 2. Search strategy for the systematic review and metaanalysis. Complete Search Strategy for a) Embase (1947 to 2020 April 08) b) Ovid MEDLINE, MEDLINE Daily and Epub Ahead of Print, In-Process \& Other Non-Indexed Citations (1947 to 2020 April 08) c) EBM Reviews Cochrane Central Register of Controlled Trials (1947 to April 2020).

Additional file 3. Excluded full text studies, with reasons. A list of full text studies excluded after screening with reasons for removal.

Additional file 4. Newcastle-Ottawa Scale for quality assessment and publication bias. A breakdown of NOS scores assigned to each study included in the meta-analysis.

Additional file 5. Forest plots for preeclampsia and funnel plots. Forest plots comparing preeclampsia in IVF/ICSI pregnancies and spontaneous pregnancies and funnel plots for publication bias in meta-analyses with 10 or more studies.

Additional file 6. Sensitivity analysis. A list of highest and lowest overall odds ratios after removing individual studies.

\section{Acknowledgements}

The authors would like to thank Sandra Mckeown from Queen's University for assisting with the search strategy for the study.

\section{Authors' contributions}

HC: conception of study design, screening, data collection and analysis, manuscript draft and revision. FTSE: conception of study design, screening, data collection, manuscript revision. LG: manuscript revision. MPV: conception of study design, supervision, manuscript revision, and approval of the version to be published. All authors approved the final version of the manuscript.

\section{Funding}

This study was supported by the Canadian Institutes of Health Research Institute of Human Development, Child and Youth Health (IHDCYH), Clinician Investigators Program, Grant number MFM-146444 and the Department of Obstetrics and Gynecology, Queen's University. The funding bodies had no role in the design of the study or the collection, analysis, and interpretation of data.

\section{Availability of data and materials}

The data underlying this article will be shared on reasonable request to the corresponding author.

\section{Declarations}

Ethics approval and consent to participate

Not applicable.

\section{Consent for publication}

Not applicable.

\section{Competing interests}

The authors declare that they have no competing interests.

\section{Author details}

'Department of Obstetrics and Gynecology, Kingston General Hospital, Queen's University, Victory 476 Stuart St, Kingston, Ontario K7L 2V7, Canada. ${ }^{2}$ Health Technology Assessment Program, Oswaldo Cruz Foundation, Av. Brasil, 4365 - Manguinhos, Rio de Janeiro, RJ 21040-900, Brazil. ${ }^{3}$ Department of Public Health Sciences, Queen's University, 62 Fifth Field Company Lane, Kingston, Ontario K7L 3N6, Canada.
Received: 10 April 2021 Accepted: 7 June 2021

Published online: 28 June 2021

\section{References}

1. Mascarenhas MN, Flaxman SR, Boerma T, Vanderpoel S, Stevens GA. National, regional, and global trends in infertility prevalence since 1990: a systematic analysis of 277 health surveys. PLoS Med. 2012;9(12):e1001356. https://doi.org/10.1371/journal.pmed.1001356.

2. Pinborg A. Short- and long-term outcomes in children born after assisted reproductive technology. Bjog. 2019;126(2):145-8. https://doi.org/10.1111/14 71-0528.15437.

3. Hamberger L, Lundin K, Sjögren A, Söderlund B. Indications for intracytoplasmic sperm injection. Hum Reprod. 1998;13(Suppl 1):128-33. https://doi.org/10.1093/humrep/13.suppl_1.128.

4. Paulson RJ. Introduction: frozen 2: an update on cryopreserved embryo transfer in the era of vitrification. Fertil Steril. 2020;113(2):239-40. https://doi. org/10.1016/j.fertnstert.2019.12.005.

5. Masoudian P, Nasr A, de Nanassy J, Fung-Kee-Fung K, Bainbridge SA, El Demellawy $\mathrm{D}$. Oocyte donation pregnancies and the risk of preeclampsia or gestational hypertension: a systematic review and metaanalysis. Am J Obstet Gynecol. 2016;214(3):328-39. https://doi.org/10.1016/j.ajog.2015.11.02

6. Jackson RA, Gibson KA, Wu YW, Croughan MS. Perinatal outcomes in singletons following in vitro fertilization: a meta-analysis. Obstet Gynecol. 2004;103(3):551-63. https://doi.org/10.1097/01.AOG.0000114989.84822.51.

7. Pandey S, Shetty A, Hamilton M, Bhattacharya S, Maheshwari A. Obstetric and perinatal outcomes in singleton pregnancies resulting from IVF/ICSI: a systematic review and meta-analysis. Hum Reprod Update. 2012;18(5):485503. https://doi.org/10.1093/humupd/dms018.

8. Stern JE, Luke B, Tobias M, Gopal D, Hornstein MD, Diop H. Adverse pregnancy and birth outcomes associated with underlying diagnosis with and without assisted reproductive technology treatment. Fertil Steril. 2015; 103(6):1438-45. https://doi.org/10.1016/j.fertnstert.2015.02.027.

9. Qin J, Wang $H$, Sheng X, Liang D, Tan H, Xia J. Pregnancy-related complications and adverse pregnancy outcomes in multiple pregnancies resulting from assisted reproductive technology: a meta-analysis of cohort studies. Fertil Steril. 2015;103(6):1492-7. https://doi.org/10.1016/j.fertnstert.2 015.03.018.

10. Thomopoulos C, Salamalekis G, Kintis K, Andrianopoulou I, Michalopoulou H, Skalis $\mathrm{G}$, et al. Risk of hypertensive disorders in pregnancy following assisted reproductive technology: overview and meta-analysis. J Clin Hypertension (Greenwich, Conn). 2017:19(2):173-83.

11. Beckmann C, Ling F, Herbert W, Laube D, Smith R. Obstetrics and gynecology. 7th edition ed. Philadelphia: Lippincott Williams \& Wilkins; 2014

12. Butalia S, Audibert F, Côté AM, Firoz T, Logan AG, Magee LA, et al. Hypertension Canada's 2018 Guidelines for the Management of Hypertension in Pregnancy. Can J Cardiol. 2018;34(5):526-31.

13. Almasi-Hashiani A, Omani-Samani R, Mohammadi M, Amini P, Navid B, Alizadeh A, et al. Assisted reproductive technology and the risk of preeclampsia: an updated systematic review and meta-analysis. BMC Pregnancy Childbirth. 2019;19(1):149. https://doi.org/10.1186/s12884-019-22 91-x.

14. Qin J, Liu X, Sheng X, Wang H, Gao S. Assisted reproductive technology and the risk of pregnancy-related complications and adverse pregnancy outcomes in singleton pregnancies: a meta-analysis of cohort studies. Fertil Steril. 2016;105(1):73-85e6. https://doi.org/10.1016/j.fertnstert.2015.09.007.

15. Helmerhorst FM, Perquin DAM, Donker D, Keirse MJNC. Perinatal outcome of singletons and twins after assisted conception: a systematic review of controlled studies. BMJ. 2004;328(7434):261.

16. Okun N, Sierra S. Pregnancy outcomes after assisted human reproduction. Obstet Gynaecol Can. 2014;36(1):64-83. https://doi.org/10.1016/\$1701-2163 (15)30685-X.

17. Roque M, Valle M, Sampaio M, Geber S. Obstetric outcomes after fresh versus frozen-thawed embryo transfers: a systematic review and metaanalysis. JBRA Assisted Reprod. 2018;22(3):253-60. https://doi.org/10.5935/1 518-0557.20180049.

18. Magee LA, Pels A, Helewa M, Rey E, von Dadelszen P. Diagnosis, evaluation, and management of the hypertensive disorders of pregnancy: executive summary. J Obstet Gynaecol Can. 2014;36(5):416-41. https://doi.org/10.101 6/S1701-2163(15)30588-0. 
19. Wells G, Shea B, O'Connell D, Peterson J, Welch V, Losos M, et al. The Newcastle-Ottawa scale (NOS) for assessing the quality of nonrandomised studies in meta-analyses. 2013.

20. Straus SE. Individualizing treatment decisions. The likelihood of being helped or harmed. Eval Health Prof. 2002;25(2):210-24. https://doi.org/10.11 77/016327870202500206

21. Patsopoulos NA, Evangelou E, loannidis JPA. Sensitivity of between-study heterogeneity in meta-analysis: proposed metrics and empirical evaluation. International Journal of Epidemiology. 2008;37:1148-57.

22. Higgins JP, Thompson SG. Quantifying heterogeneity in a meta-analysis. Statistics in medicine. 2002;21:1539-58.

23. Sterne JA, Sutton AJ, loannidis JP, Terrin N, Jones DR, Lau J, et al. Recommendations for examining and interpreting funnel plot asymmetry in meta-analyses of randomised controlled trials. BMJ (Clinical research ed). 2011;343.

24. Agarwal P, Loh S, Lim S, Sriram B, Daniel M, Yeo S, et al. Two-year neurodevelopmental outcome in children conceived by intracytoplasmic sperm injection: prospective cohort study. BJOG. 2005;112(10):1376-83.

25. Ai W, Liu Z. Study on the adverse outcomes in twins conceived by IVF-ET. Matern Child Health Care China. 2005;23:3119-20.

26. Apantaku O, Chandrasekaran I, Bentick B. Obstetric outcome of singleton pregnancies achieved with in vitro fertilisation and intracytoplasmic sperm injection: experience from a district general hospital. J Obstetrics Gynaecol. 2008;28(4):398-402. https://doi.org/10.1 080/01443610802091792.

27. Aydin CA, Aydin S, Serdaroglu H. Multifetal gestations with assisted reproductive technique before the single-embryo transfer legislation: obstetric, neonatal outcomes and congenital anomalies. J Maternal-fetal Neonatal Med. 2016;29(15):2475-80.

28. Barda G, Gluck O, Mizrachi Y, Bar J. A comparison of maternal and perinatal outcome between in vitro fertilization and spontaneous dichorionic-diamniotic twin pregnancies. J Maternal-fetal Neonatal Med. 2017;30(24):2974-7. https://doi.org/10.1080/14767058.2016.1270934.

29. Barua S, Hng TM, Smith H, Bradford J, Mclean M. Ovulatory disorders are an independent risk factor for pregnancy complications in women receiving assisted reproduction treatments. The Australian \& New Zealand journal of obstetrics \& gynaecology. 2016;57(3):286-93

30. Beltran AA, Pauly $V$, Riviere $O$, Sambuc R, Boyer $P$, Vendittelli $F$, et al. Birthweight of IVF children is still a current issue and still related to maternal factors. Reprod BioMed Online. 2019;39(6):990-9. https://doi.org/10.1016/j. rbmo.2019.09.014.

31. Bensdorp AJ, Hukkelhoven CW, van der Veen F, Mol BWJ, Lambalk CB, van Wely M. Dizygotic twin pregnancies after medically assisted reproduction and after natural conception: maternal and perinatal outcomes. Fertil Steril. 2016;106(2):371-7 e2.

32. Beyer DA, Amari F. Maternal risk factors and neonatal outcomes after ART treatment - a German monocenter experience. Middle East Fertil Soc J. 2016;21(3):155-60. https://doi.org/10.1016/j.mefs.2015.12.001.

33. Carbone IF, Cruz JJ, Sarquis R, Akolekar R, Nicolaides KH. Assisted conception and placental perfusion assessed by uterine artery Doppler at 11-13 weeks' gestation. Human reproduction. 2011;26(7):1659-64.

34. Caserta D, Marci R, Tatone C, Schimberni M, Vaquero E, Lazzarin N, et al. IVF pregnancies: neonatal outcomes after the new Italian law on assisted reproduction technology (law 40/2004). Acta Obstet Gynecol Scand. 2008; 87(9):935-9. https://doi.org/10.1080/00016340802283681.

35. Caserta D, Bordi G, Stegagno M, Filippini F, Podagrosi M, Roselli D, et al. Maternal and perinatal outcomes in spontaneous versus assisted conception twin pregnancies. Eur J Obstet Gynecol Reprod Biol. 2014; 174(64-69).

36. Choi SJ, Kim HS, Roh CR. Pregnancy outcomes of twins after in vitro and spontaneous fertilization. Int J Gynecol Obstet. 2006;94(1):49-51. https://doi. org/10.1016/j.ijgo.2006.04.015

37. Daniel Y, Ochshorn Y, Fait G, Geva E, Bar-Am A, Lessing JB. Analysis of 104 twin pregnancies conceived with assisted reproductive technologies and 193 spontaneously conceived twin pregnancies. Fertil Steril. 2000;74(4):6839. https://doi.org/10.1016/S0015-0282(00)01491-6.

38. Dayan N, Pilote L, Opatrny L, Basso O, Messerlian C, El-Messidi A, et al. Combined impact of high body mass index and in vitro fertilization on preeclampsia risk: a hospital-based cohort study. Obesity (Silver Spring, Md). 2015;23(1):200-6.
39. Dayan N, Lanes A, Walker MC, Spitzer KA, Laskin CA. Effect of chronic hypertension on assisted pregnancy outcomes: a population-based study in Ontario, Canada. Fertil Steril. 2016;105(4):1003-9. https://doi.org/10.1016/j. fertnstert.2015.11.039.

40. Dayan N, Fell DB, Guo Y, Wang H, Velez MP, Spitzer K, et al. Severe maternal morbidity in women with high BMI in IVF and unassisted singleton pregnancies. Hum Reprod (Oxford, England). 2018;33(8):1548-56.

41. Deltombe-Bodart S, Deruelle P, Drumez E, Cordiez S, Catteau-Jonard S, Garabedian C. Obstetrical and perinatal complications of twin pregnancies: is there a link with the type of infertility treatment? Acta Obstet Gynecol Scand. 2017;96(7):844-51. https://doi.org/10.1111/aogs.13135.

42. Dior UP, Laufer N, Chill HH, Granovsky-Grisaru S, Yagel S, Yaffe H, et al. Increased incidence of preeclampsia in mothers of advanced age conceiving by oocyte donation. Arch Gynecol Obstet. 2018;297(5):1293-9. https://doi.org/10.1007/s00404-017-4616-1.

43. Elenis E, Svanberg AS, Lampic C, Skalkidou A, Akerud H, Sydsjo G. Adverse obstetric outcomes in pregnancies resulting from oocyte donation: a retrospective cohort case study in Sweden. BMC Pregnancy Childbirth. 2015; 15(100967799):247. https://doi.org/10.1186/s12884-015-0687-9.

44. Ernstad EG, Wennerholm U-B, Khatibi A, Petzold M, Bergh C. Neonatal and maternal outcome after frozen embryo transfer: Increased risks in programmed cycles. Am J Obstet Gynecol. 2019;221(2):126 e1-.e18.

45. Fan C, Sun Y, Yang J, Ye J, Wang S. Maternal and neonatal outcomes in dichorionic twin pregnancies following IVF treatment: a hospital-based comparative study. International journal of clinical and experimental pathology. 2013;6(10):2199-207.

46. Farhi A, Reichman B, Boyko V, Hourvitz A, Ron-El R, Lerner-Geva L. Maternal and neonatal health outcomes following assisted reproduction. Reprod BioMed Online. 2013;26(5):454-61. https://doi.org/10.1016/j.rbmo.2013.01. 014.

47. Geipel A, Ludwig M, Germer U, Katalinic A, Diedrich K, Gembruch U. Uterine artery Doppler velocimetry and the outcome of pregnancies resulting from ICSI. Human reproduction. 2001;16(7):1397-402.

48. Gocmen A, Guven S, Bagci S, Cekmez Y, Sanlikan F. Comparison of maternal and fetal outcomes of IVF and spontaneously conceived twin pregnancies: three year experience of a tertiary hospital. Int J Clin Exp Med. 2015;8(4): 6272-6.

49. Gojnic M, Jeremic K, Boskovic V, Fazlagic A, Stefanovic A, Pervulov M. Perinatal outcome in multiple pregnancies - spontaneous gestation versus. Clin Exp Obstet Gynecol. 2005;32(1):65-7.

50. Hessami K, Kasraeian M, Shoushtari S, Hessami A. Maternal and Neonatal Outcomes of Monochorionic and Dichorionic Twin Pregnancies Following Assisted Reproductive Technology in Southern Iranian Women. Shiraz EMed J. 2020;21(3)

51. Howe RS, Sayegh RA, Durinzi KL, Tureck RW. Perinatal outcome of singleton pregnancies conceived by in vitro fertilization: a controlled study. Journal of perinatology : official journal of the California Perinatal Association. 1990; 10(3):261-6.

52. Jancar N, Mihevc Ponikvar B, Tomsic S, Vrtacnik Bokal E, Korosec S. IS IVF/ISCI an Independent Risk Factor for Spontaneous Preterm Birth in Singletons? A Population-Based Cohort Study. BioMed Research International. 2018; 7124362.

53. Jeve YB, Potdar N, Opoku A, Khare M. Three-arm age-matched retrospective cohort study of obstetric outcomes of donor oocyte pregnancies. Int J Gynaecol Obstetrics. 2016;133(2):156-8. https://doi.org/10.1016/j.ijgo.2015.09.024.

54. Katalinic A, Rösch C, Ludwig M, Group. GIF-US. Pregnancy course and outcome after intracytoplasmic sperm injection: a controlled, prospective cohort study. Fertility and sterility. 2004;81(6):1604-16.

55. Koivurova S, Hartikainen AL, Karinen L, Gissler M, Hemminki E, Martikainen $\mathrm{H}$, et al. The course of pregnancy and delivery and the use of maternal healthcare services after standard IVF in northern Finland 1990-1995. Hum Reprod. 2002;17(11):2897-903. https://doi.org/10.1093/humrep/17.11.2897.

56. Korosec S, Frangez HB, Steblovnik L, Verdenik I, Bokal EV. Independent factors influencing large-for-gestation birth weight in singletons born after in vitro fertilization. J Assist Reprod Genet. 2016;33(1):9-17. https://doi.org/1 0.1007/s10815-015-0601-4

57. Kouhkan A, Khamseh ME, Pirjani R, Moini A, Arabipoor A, Maroufizadeh S, et al. Obstetric and perinatal outcomes of singleton pregnancies conceived via assisted reproductive technology complicated by gestational diabetes mellitus: a prospective cohort study. BMC Pregnancy Childbirth. 2018;18(1): 495. https://doi.org/10.1186/s12884-018-2115-4. 
58. Kuivasaari-Pirinen P, Raatikainen K, Hippelainen M, Heinonen S. Adverse outcomes of IVF/ICSI pregnancies vary depending on aetiology of infertility. ISRN Obstetrics and Gynecology. 2012;(451915).

59. Lee MS, Cantonwine D, Little SE, McElrath TF, Parry SI, Lim K-H, et al. Angiogenic markers in pregnancies conceived through in vitro fertilization. Am J Obstet Gynecol. 2015:213(2):212 e1-8.

60. Lei L-L, Lan Y-L, Wang S-Y, Feng W, Zhai Z-J. Perinatal complications and live-birth outcomes following assisted reproductive technology: a retrospective cohort study. Chin Med J. 2019;132(20):2408-16. https://doi. org/10.1097/CM9.0000000000000484.

61. Li J, Yang J, Xu WM, Cheng D, Zou YJ. Comparison of the perinatal outcome of twins conceived after assisted reproductive technologies versus those conceived naturally. J Reprod Med. 2015;60(1):37-42.

62. Luke B, Brown MB, Wantman E, Seifer DB, Sparks AT, Lin PC, et al. Risk of prematurity and infant morbidity and mortality by maternal fertility status and plurality. J Assist Reprod Genet. 2019;36(1):121-38. https://doi.org/10.1 007/s10815-018-1333-z.

63. Luke B, Brown MB, Eisenberg ML, Callan C, Botting BJ, Pacey A, et al. In vitro fertilization and risk for hypertensive disorders of pregnancy: associations with treatment parameters. Am J Obstet Gynecol. 2020;222(4):350 e1-.e13.

64. Malchau SS, Loft A, Larsen EC, Aaris Henningsen A-K, Rasmussen S, Andersen AN, et al. Perinatal outcomes in 375 children born after oocyte donation: a Danish national cohort study. Fertil Steril. 2013;99(6):1637-43. https://doi.org/10.1016/j.fertnstert.2013.01.128.

65. Martínez-Varea A, Pellicer B, Serra V, Hervás-Marín D, Martínez-Romero A, Bellver J, et al. The Maternal Cytokine and Chemokine Profile of Naturally Conceived Gestations Is Mainly Preserved during In Vitro Fertilization and Egg Donation Pregnancies. Journal of immunology research. 2015;128616.

66. Meyer R, Orvieto R, Timerman Y, Gorodesky T, Toussia-Cohen S, Kedem A, et al. Impact of the mode of conception on gestational hypertensive disorders at very advanced maternal age. Reprod BioMed Online. 2020;40(2): 281-6. https://doi.org/10.1016/j.rbmo.2019.10.017.

67. Mohammed A-BF, Abdel-Maaboud M. Obstetric and neonatal outcomes of IVF versus spontaneously conceived dichorionic twins. Middle East Fertil Soc J. 2012;17(4):231-5. https://doi.org/10.1016/j.mefs.2012.02.008.

68. Moini A, Shiva M, Arabipoor A, Hosseini R, Chehrazi M, Sadeghi M. Obstetric and neonatal outcomes of twin pregnancies conceived by assisted reproductive technology compared with twin pregnancies conceived spontaneously: a prospective follow-up study. European journal of obstetrics, gynecology, and reproductive biology. 2012;165(1):29-32.

69. Nagata C, Yang L, Yamamoto-Hanada K, Mezawa H, Ayabe T, Ishizuka K, et al. Complications and adverse outcomes in pregnancy and childbirth among women who conceived by assisted reproductive technologies: a nationwide birth cohort study of Japan environment and children's study. BMC Pregnancy Childbirth. 2019;19(1):77. https://doi.org/10.1186/s12884-019-2213-y.

70. Nassar AH, Usta IM, Rechdan JB, Harb TS, Adra AM, Abu-Musa AA. Pregnancy outcome in spontaneous twins versus twins who were conceived through in vitro fertilization. Am J Obstet Gynecol. 2003;189(2): 513-8. https://doi.org/10.1067/S0002-9378(03)00485-X.

71. Nejdet S, Bergh C, Kallen K, Wennerholm U-B, Thurin-Kjellberg A. High risks of maternal and perinatal complications in singletons born after oocyte donation. Acta Obstet Gynecol Scand. 2016;95(8):879-86. https://doi.org/1 0.1111 /aogs. 12904

72. Ochsenkühn R, Strowitzki T, Gurtner M, Strauss A, Schulze A, Hepp H, et al. Pregnancy complications, obstetric risks, and neonatal outcome in singleton and twin pregnancies after GIFT and IVF. Archives of gynecology and obstetrics. 2003:268(4):256-61

73. Okby R, Harlev A, Sacks KN, Sergienko R, Sheiner E. Preeclampsia acts differently in in vitro fertilization versus spontaneous twins. Arch Gynecol Obstet. 2018;297(3):653-8. https://doi.org/10.1007/s00404-017-4635-y.

74. Olivennes F, Rufat P, André B, Pourade A, Quiros MC, Frydman R. The increased risk of complication observed in singleton pregnancies resulting from in-vitro fertilization (IVF) does not seem to be related to the IVF method itself. Human reproduction. 1993(8):1297-300.

75. Opdahl S, Henningsen AA, Tiitinen A, Bergh C, Pinborg A, Romundstad PR, et al. Risk of hypertensive disorders in pregnancies following assisted reproductive technology: a cohort study from the CoNARTaS group. Hum Reprod (Oxford, England). 2015;30(7):1724-31.

76. Poikkeus P, Gissler M, Unkila-Kallio L, Hyden-Granskog C, Tiitinen A. Obstetric and neonatal outcome after single embryo transfer. Hum Reprod (Oxford, England). 2007;22(4):1073-9.
77. Qin J, Sheng X, Wu D, Gao S, You Y, Yang T, et al. Adverse Obstetric Outcomes Associated With In Vitro Fertilization in Singleton Pregnancies. Reprod Sci (Thousand Oaks, Calif). 2017;24(4):595-608.

78. Raisanen S, Randell K, Nielsen HS, Gissler M, Kramer MR, Klemetti R, et al. Socioeconomic status affects the prevalence, but not the perinatal outcomes, of in vitro fertilization pregnancies. Hum Reprod (Oxford, England). 2013;28(11):3118-25.

79. Reismullerova L, Holoman K, Polackova-Borosova M, Luha J. Polycystic ovary syndrome - a risk factor of pre-eclampsia after in vitro fertilisation. Bratisl Lek Listy. 2015;116(5):311-5. https://doi.org/10.4149/bIl_2015_058.

80. Reubinoff B, Samueloff A, Ben-Haim M, Friedler S, Schenker JLA. Is the obstetric outcome of in vitro fertilized singleton gestations different from natural ones? A controlled study. Fertility and sterility. 1997;67(6):1077-83.

81. Rizzo G, Aiello E, Pietrolucci ME, Arduini D. Are There Differences in Placental Volume and Uterine Artery Doppler in Pregnancies Resulting From the Transfer of Fresh Versus Frozen-Thawed Embryos Through In Vitro Fertilization. Reproductive sciences. 2016;23(10):1381-6.

82. Rizzo G, Aiello E, Pietrolucci ME, Arduini D. Placental volume and uterine artery Doppler evaluation at $11+0$ to $13+6$ weeks' gestation in pregnancies conceived with in-vitro fertilization: comparison between autologous and donor oocyte recipients. Ultrasound Obstetrics Gynecol. 2016;47(6):726-31. https://doi.org/10.1002/uog.14918.

83. Sazonova A, Kallen K, Thurin-Kjellberg A, Wennerholm U-B, Bergh C. Obstetric outcome in singletons after in vitro fertilization with cryopreserved/thawed embryos. Hum Reprod (Oxford, England). 2012;27(5):1343-50.

84. Shi J, Zhang Y, Luo H, Han Y, Du A. Clinical outcomes of test-tube versus naturally-conceived twins. Int J Clin Exp Med. 2018;11(3):2507-12.

85. Shiloh SR, Sheiner E, Wainstock T, Walfisch A, Segal I, Landau D, et al. Longterm cardiovascular morbidity in children born following fertility treatment. J Pediatr. 2019;204:84-8. https://doi.org/10.1016/j.jpeds.2018.08.070.

86. Silberstein T, Levy A, Harlev A, Saphier O, Sheiner E. Perinatal outcome of pregnancies following in vitro fertilization and ovulation induction. J Maternal-fetal Neonatal Med. 2014;27(13):1316-9. https://doi.org/10.3109/14 767058.2013.856415.

87. Stojnic J, Radunovic N, Jeremic K, Kotlica BK, Mitrovic M, Tulic I. Perinatal outcome of singleton pregnancies following in vitro fertilization. Clinical and experimental obstetrics \& gynecology. 2013;40(2):277-83.

88. Sun L-M, Walker MC, Cao H-L, Yang Q, Duan T, Kingdom JCP. Assisted reproductive technology and placenta-mediated adverse pregnancy outcomes. Obstet Gynecol. 2009;114(4):818-24. https://doi.org/10.1097/AOG. Ob013e3181b76bd1.

89. Sun L, Zou G, Wei X, Chen Y, Zhang J, Okun N, et al. Clinical outcomes after assisted reproductive technology in twin pregnancies: chorionicity-based comparison. Scientific reports. 2016;6(26869).

90. Suzuki S, Miyake H. Perinatal outcomes of elderly primiparous dichorionic twin pregnancies conceived by in vitro fertilization compared with those conceived spontaneously. Arch Gynecol Obstet. 2010;281(1):87-90. https:// doi.org/10.1007/s00404-009-1083-3.

91. Szymusik I, Kosinska-Kaczynska K, Bomba-Opon D, Wielgos M. IVF versus spontaneous twin pregnancies - which are at higher risk of complications. J Matern Fetal Neonatal Med. 2012;25(12):2725-8. https://doi.org/10.3109/14 767058.2012 .705398 .

92. Szymusik I, Kosinski P, Kosinska-Kaczynska K, Warzecha D, Karwacka A, Kaczynski B, et al. The first trimester aneuploidy biochemical markers in IVF/ ICSI patients have no additional benefit compared to spontaneous conceptions in the prediction of pregnancy complications. J Perinat Med. 2018;46(9):953-9. https://doi.org/10.1515/.jpm-2017-0199.

93. Tan SL, Doyle P, Campbell S, Beral V, Rizk B, Brinsden P, et al. Obstetric outcome of in vitro fertilization pregnancies compared with normally conceived pregnancies. American journal of obstetrics and gynecology. 1992;167(3):778-84.

94. Tandberg A, Klungsoyr K, Romundstad LB, Skjaerven R. Pre-eclampsia and assisted reproductive technologies: consequences of advanced maternal age, interbirth intervals, new partner and smoking habits. BJOG. 2015;122(7): 915-22. https://doi.org/10.1111/1471-0528.13051.

95. Tomic V, Tomic J. Neonatal outcome of IVF singletons versus naturally conceived in women aged 35 years and over. Archives of gynecology and obstetrics. 2011;284(6):1411-6

96. Valenzuela-Alcaraz B, Crispi F, Bijnens B, Cruz-Lemini M, Creus M, Sitges M, et al. Assisted reproductive technologies are associated with cardiovascular 
remodeling in utero that persists postnatally. Circulation. 2013;128(13):144250. https://doi.org/10.1161/CIRCULATIONAHA.113.002428.

97. Valenzuela-Alcaraz BA-O, Cruz-Lemini M, Rodríguez-López M, Goncé A, García-Otero L, Ayuso H, et al. Fetal cardiac remodeling in twin pregnancy conceived by assisted reproductive technology. Ultrasound Obstetrics Gynecol. 2018:51(1):94-100. https://doi.org/10.1002/uog.17527.

98. Vasario E, Borgarello V, Bossotti C, Libanori E, Biolcati M, Arduino S, et al. IVF twins have similar obstetric and neonatal outcome as spontaneously conceived twins: a prospective follow-up study. Reproductive biomedicine online. 2012;21(3):422-8.

99. von Versen-Höynck F, Schaub AM, Chi YY, Chiu KH, Liu J, Lingis M, et al. Increased preeclampsia risk and reduced aortic compliance with in vitro fertilization cycles in the absence of a Corpus luteum. Hypertension. 2019; 73(3):640-9. https://doi.org/10.1161/HYPERTENSIONAHA.118.12043.

100. Watanabe N, Fujiwara T, Suzuki T, Jwa SC, Taniguchi K, Yamanobe Y, et al. Is in vitro fertilization associated with preeclampsia? A propensity score matched study. BMC Pregnancy Childbirth. 2014;14(100967799):69. https:// doi.org/10.1186/1471-2393-14-69.

101. Wennberg AL, Opdahl S, Bergh C, Aaris Henningsen A-K, Gissler M, Romundstad $L B$, et al. Effect of maternal age on maternal and neonatal outcomes after assisted reproductive technology. Fertil Steril. 2016;106(5): $1142-9$ e14

102. Wu QF, Xu DF, Zhao Y, Xin CL. Outcomes of 223 twins born after assisted reproductive techniques compared with 281 twins born after natural conception. Jiangxi Med J. 2010;46:95-7.

103. Xu Z, Ye B, Lin J, Lin W, Lin L. Analysis of perinatal outcomes of twin pregnancies conceived by in vitro fertilization and embryo transfer (IVF-ET) and those conceived spontaneously. J Wenzhou Med Coll. 2005;35:34-6.

104. Yang H, Choi YS, Nam KH, Kwon JY, Park YW, Kim YH. Obstetric and perinatal outcomes of dichorionic twin pregnancies according to methods of conception: spontaneous versus in-vitro fertilization. Twin Res Hum Genetics. 2011;14(1):98-103. https://doi.org/10.1375/twin.14.1.98.

105. Yang X, Li Y, Li C, Zhang W. Current overview of pregnancy complications and live-birth outcome of assisted reproductive technology in mainland China. Fertil Steril. 2014;101 (2):385-91. https://doi.org/10.1016/j.fertnstert.2 013.10.017.

106. Zádori J, Kozinszky Z, Orvos H, Katona M, Pál A, Kovács L. Dilemma of increased obstetric risk in pregnancies following IVF-ET. Journal of assisted reproduction and genetics. 2003;20(6):216-21.

107. Zhang H-Q, Fan R, Tian G-H, Li L, Zhang Z-H, Zhang Y-P. Clinical outcomes of twin pregnancies conceived by in vitro fertilization compared with spontaneous twin pregnancies. Zhongguo Dang Dai Er Ke Za Zhi. 2015; 17(1):63-7.

108. Zhu L, Zhang Y, Liu Y, Zhang R, Wu Y, Huang Y, et al. Maternal and livebirth outcomes of pregnancies following assisted reproductive technology: a retrospective cohort study. Sci Rep. 2016;6(101563288):35141. https://doi. org/10.1038/srep35141.

109. Lalani S, Choudhry AJ, Firth B, Bacal V, Walker M, Wen SW, et al. Endometriosis and adverse maternal, fetal and neonatal outcomes, a systematic review and meta-analysis. Hum Reprod (Oxford, England). 2018; 33(10):1854-65.

110. Bosch E, De Vos M, Humaidan P. The Future of Cryopreservation in Assisted Reproductive Technologies. Front Endocrinol (Lausanne). 2020;11:67.

111. Maheshwari A, Pandey S, Raja EA, Shetty A, Hamilton M, Bhattacharya S. Is frozen embryo transfer better for mothers and babies? Can cumulative meta-analysis provide a definitive answer? Hum Reprod Update. 2018;24(1): 35-58. https://doi.org/10.1093/humupd/dm×031.

112. Sha T, Wang X, Cheng W, Yan Y. A meta-analysis of pregnancy-related outcomes and complications in women with polycystic ovary syndrome undergoing IVF. Reprod BioMed Online. 2019;39(2):281-93. https://doi.org/1 0.1016/j.rbmo.2019.03.203.

113. von Versen-Höynck F, Narasimhan P, Selamet Tierney ES, Martinez N, Conrad KP, Baker VL, et al. Absent or excessive Corpus luteum number is associated with altered maternal vascular health in early pregnancy. Hypertension. 2019;73(3):680-90. https://doi.org/10.1161/HYPERTENSIONA HA. 118.12046

114. von Versen-Höynck F, Strauch NK, Liu J, Chi YY, Keller-Woods M, Conrad KP, et al. Effect of mode of conception on maternal serum Relaxin, creatinine, and sodium concentrations in an infertile population. Reprod Sci. 2019;26(3): 412-9. https://doi.org/10.1177/1933719118776792.
115. Luke B, Brown MB, Eisenberg ML, Callan C, Botting BJ, Pacey A, et al. In Vitro Fertilization and Risk for Hypertensive Disorders of Pregnancy: Associations with Treatment Parameters. American journal of obstetrics and gynecology. 2019:222(4):350.e1-.e13.

116. Elias FTS, Weber-Adrian D, Pudwell J, Carter J, Walker M, Gaudet L, et al. Neonatal outcomes in singleton pregnancies conceived by fresh or frozen embryo transfer compared to spontaneous conceptions: a systematic review and meta-analysis. Arch Gynecol Obstet. 2020;302(1):31-45. https:// doi.org/10.1007/s00404-020-05593-4.

117. Sauer MV. Revisiting the early days of oocyte and embryo donation: relevance to contemporary clinical practice. Fertil Steril. 2018;110(6):981-7. https://doi.org/10.1016/j.fertnstert.2018.09.005.

118. Kupka MS, Ferraretti AP, de Mouzon J, Erb K, D'Hooghe T, Castilla JA, et al. Assisted reproductive technology in Europe, 2010: results generated from European registers by ESHRE†. Hum Reprod. 2014;29(10):2099-113. https:// doi.org/10.1093/humrep/deu175.

119. Storgaard M, Loft A, Bergh C, Wennerholm UB, Soderstrom-Anttila V, Romundstad LB, et al. Obstetric and neonatal complications in pregnancies conceived after oocyte donation: a systematic review and meta-analysis. BJOG. 2017;124(4):561-72. https://doi.org/10.1111/1471-0528.14257.

120. Pecks U, Maass N, Neulen J. Oocyte donation: a risk factor for pregnancyinduced hypertension: a meta-analysis and case series. Deutsches Arzteblatt international. 2011;108(3):23-31.

121. Levron Y, Dviri M, Segol I, Yerushalmi GM, Hourvitz A, Orvieto R, et al. The 'immunologic theory' of preeclampsia revisited: a lesson from donor oocyte gestations. Am J Obstet Gynecol. 2014;211(4):383 e1-5.

122. Tubbergen P, Lachmeijer AM, Althuisius SM, Vlak ME, van Geijn HP, Dekker GA. Change in paternity: a risk factor for preeclampsia in multiparous women? Journal of reproductive immunology. 1999;45(1):81-8.

123. Lashley LE, Haasnoot GW, Spruyt-Gerritse M, Claas FH. Selective advantage of HLA matching in successful uncomplicated oocyte donation pregnancies. J Reprod Immunol. 2015;112:29-33. https://doi.org/10.1016/j.ji.2015.05.006.

124. Velez MP, Hamel C, Hutton B, Gaudet L, Walker M, Thuku M, et al. Care plans for women pregnant using assisted reproductive technologies: a systematic review. Reprod Health. 2019;16(1):9. https://doi.org/10.1186/s12 978-019-0667-z.

125. Henderson J, Whitlock E, O'Connor E, Senger C, Thompson J, Rowland M. Low-dose aspirin for prevention of morbidity and mortality from preeclampsia: a systematic evidence review for the U.S. preventive services task force. Ann Intern Med. 2014;160(10):695-703. https://doi.org/10.7326/ M13-2844.

\section{Publisher's Note}

Springer Nature remains neutral with regard to jurisdictional claims in published maps and institutional affiliations.

Ready to submit your research? Choose BMC and benefit from:

- fast, convenient online submission

- thorough peer review by experienced researchers in your field

- rapid publication on acceptance

- support for research data, including large and complex data types

- gold Open Access which fosters wider collaboration and increased citations

- maximum visibility for your research: over $100 \mathrm{M}$ website views per year

At $\mathrm{BMC}$, research is always in progress.

Learn more biomedcentral.com/submissions 